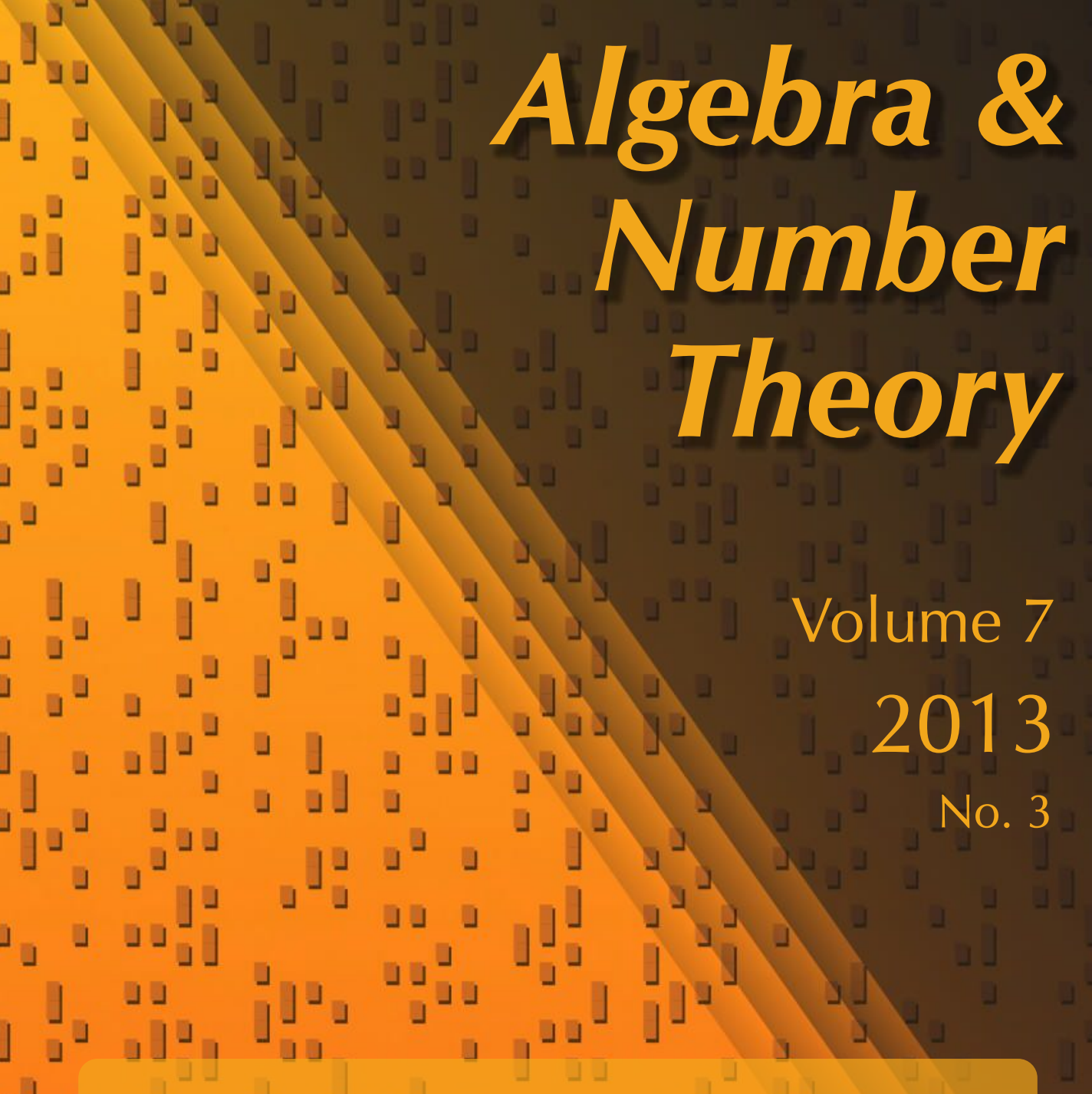

Cycle classes and the syntomic regulator Bruno Chiarellotto, Alice Ciccioni and Nicola Mazzari

」

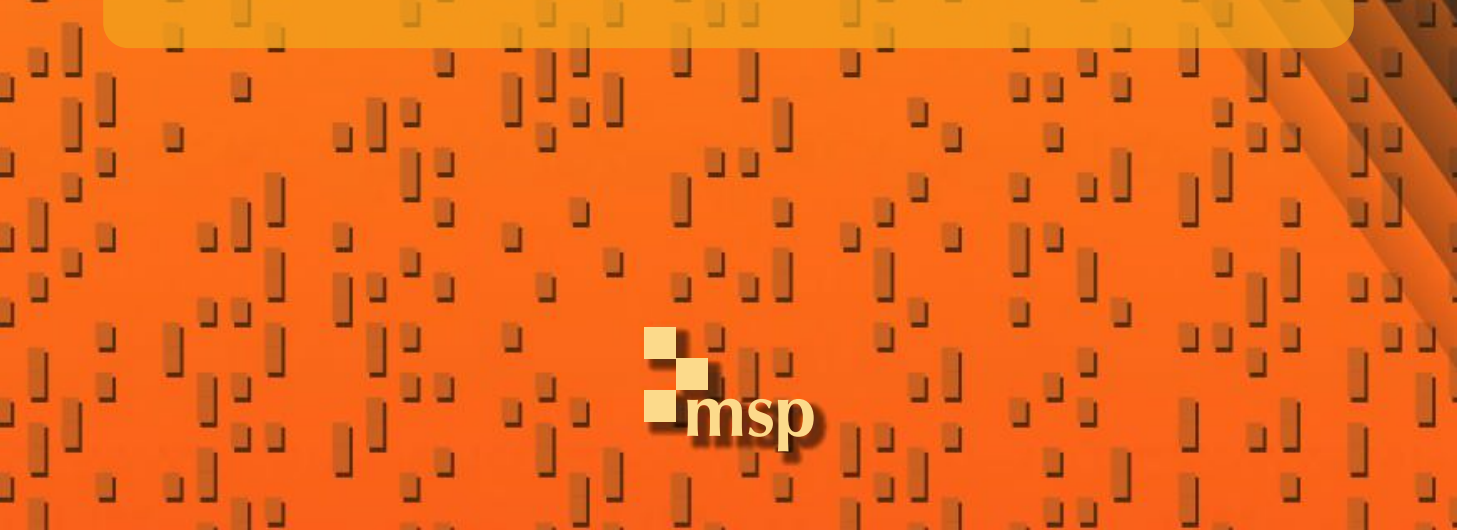




\title{
Cycle classes and the syntomic regulator
}

\author{
Bruno Chiarellotto, Alice Ciccioni and Nicola Mazzari
}

Let $\mathscr{V}=\operatorname{Spec}(R)$ and $R$ be a complete discrete valuation ring of mixed characteristic $(0, p)$. For any flat $R$-scheme $\mathscr{X}$, we prove the compatibility of the de Rham fundamental class of the generic fiber and the rigid fundamental class of the special fiber. We use this result to construct a syntomic regulator map $\operatorname{reg}_{\text {syn }}: C H^{i}(\mathscr{X} / \mathscr{V}, 2 i-n) \rightarrow H_{\text {syn }}^{n}(\mathscr{X}, i)$ when $\mathscr{X}$ is smooth over $R$ with values in the syntomic cohomology defined by A. Besser. Motivated by the previous result, we also prove some of the Bloch-Ogus axioms for the syntomic cohomology theory but viewed as an absolute cohomology theory.

\section{Introduction}

Let $\mathscr{V}=\operatorname{Spec}(R)$ with $R$ a complete discrete valuation ring of mixed characteristic and perfect residue field. Given $\mathscr{X}$, an algebraic $\mathscr{V}$-scheme, one can consider the de Rham cohomology of its generic fiber $\mathscr{X}_{K}$ and the rigid cohomology of its special fiber $\mathscr{X}_{k}$. These two cohomology groups are related by a canonical cospecialization map cosp : $H_{\text {rig, }, c}^{n}\left(\mathscr{X}_{k}\right) \rightarrow H_{\mathrm{dR}, c}^{n}\left(\mathscr{X}_{K}\right)$ (in general not an isomorphism) [Baldassarri et al. 2004, Section 6]. There is also the notion of rigid and de Rham cycle class. The starting result of this paper is the compatibility of these cycle classes with respect to the cospecialization map (see Theorem 1.4.1 for the precise statement).

In the case $\mathscr{X}$ is smooth (possibly nonproper) over $\mathscr{V}$, we get the following corollary (see Corollary 1.5.1). Let $s p_{C H}: C H^{*}\left(\mathscr{Q}_{K}\right) \otimes \mathbb{Q} \rightarrow C H^{*}\left(\mathscr{X}_{k}\right) \otimes \mathbb{Q}$ be the specialization of Chow rings constructed by Grothendieck in [Berthelot et al. 1971, Appendix]. Then the diagram

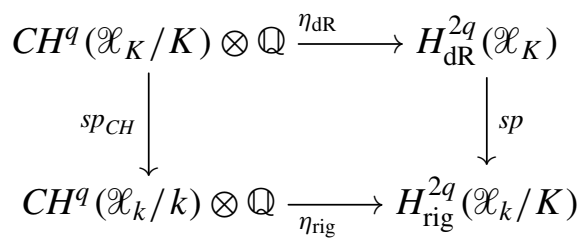

The three authors were supported by the Cariparo Eccellenza Grant "Differential Methods in Arithmetic, Geometry and Algebra".

MSC2010: primary 14F43; secondary 14F30, $19 \mathrm{~F} 27$.

Keywords: syntomic cohomology, cycles, regulator map, rigid cohomology, de Rham cohomology. 
is commutative, where $\eta_{\mathrm{dR}}$ and $\eta_{\text {rig }}$ are the de Rham and cycle class maps, respectively, and $s p$ is the Poincare dual of cosp. In the proof, we use the main results of [Baldassarri et al. 2004; Bosch et al. 1995; Petrequin 2003].

This result can be viewed as a generalization of a theorem of Messing [1987, Theorem B3.1], in which he further assumes $\mathscr{X}$ to be proper (not only smooth) over $\mathscr{V}$. In that case, rigid and crystalline coincide, and the map $s p$ is an isomorphism [Berthelot 1997b].

This compatibility result is the motivation for an alternative construction of the regulator map (see Proposition 1.6.6)

$$
\operatorname{reg}_{\text {syn }}: C H^{i}(\mathscr{X} / \mathscr{V}, 2 i-n) \rightarrow H_{\text {syn }}^{n}(\mathscr{X}, i)
$$

with values in the syntomic cohomology group defined by Besser [2000] (for $\mathscr{X}$ smooth over $\mathscr{V}$ ). For this proof, we use an argument of Bloch [1986] and the existence of a syntomic cycle class (see Proposition 1.6.2).

The aforementioned results motivated us to investigate further the properties of syntomic cohomology. We are not able to formulate even the basic Bloch-Ogus axioms using Besser's framework. Thus, we have followed the interpretation of syntomic cohomology of Bannai [2002] as an absolute one. To this end, we define a triangulated category of $p$-adic Hodge complexes, $p H D$ (see Definition 2.0.11). An object $M$ of $p H D$ can be represented by a diagram of the form $M_{\text {rig }} \rightarrow M_{K} \leftarrow M_{\mathrm{dR}}$, where $M_{\text {? }}$ is a complex of $K$-vector spaces endowed with a Frobenius automorphism when $?=$ rig and with a filtration when $?=\mathrm{dR}$. In $p H D$, there is a naturally defined tensor product, and $\mathbb{K}$ denotes the unit object of $p H D$. The main difference with respect to [Bannai 2002] is that the maps in the diagram are not necessarily quasiisomorphisms.

From [Besser 2000], we get (in Proposition 5.3.1) that there are functorial $p$-adic Hodge complexes $R \Gamma(\mathscr{C})$ satisfying

$$
R \Gamma_{\text {rig }}(\mathscr{X}) \rightarrow R \Gamma_{K}(\mathscr{X}) \leftarrow R \Gamma_{\mathrm{dR}}(\mathscr{C})
$$

and inducing the specialization map in cohomology (that is, taking the cohomology of each element of the diagram). Meanwhile, we show how the constructions made by Besser may be obtained using the theory of generalized Godement resolution (also called the bar resolution). In particular, we use the results of [van der Put and Schneider 1995] in order to have enough points for rigid analytic spaces. Further, we consider the twisted version $R \Gamma(\mathscr{X})(i)$, which is given by the same complexes but with the Frobenius (respectively the filtration) twisted by $i$ (see Remark 2.2.1).

We then prove (see Proposition 5.3.4) that the syntomic cohomology groups $H_{\text {syn }}^{n}(\mathscr{X}, i)$ of [Besser 2000] are isomorphic to the (absolute cohomology) groups $H_{\mathrm{abs}}^{n}(\mathscr{X}, i):=\operatorname{Hom}_{p H D}(\mathbb{K}, R \Gamma(\mathscr{X})(i)[n])$. This result generalizes that of [Bannai 
2002] (which was given only for $\mathscr{V}$-schemes with good compactification) to any smooth algebraic $\mathscr{V}$-scheme.

For this absolute cohomology, we can prove some of the Bloch-Ogus axioms. In fact, we construct a $p$-adic Hodge complex $R \Gamma_{c}(\mathscr{X})(i)$ related to rigid and de Rham cohomology with compact support. Therefore, we can define an absolute cohomology with compact support functorial with respect to proper maps $H_{\text {abs, } c}^{n}(\mathscr{X}, i):=\operatorname{Hom}_{p H D}\left(\mathbb{K}, R \Gamma_{c}(\mathscr{X})(i)[n]\right)$ and an absolute homology theory $H_{n}^{\mathrm{abs}}(\mathscr{X}, i):=\operatorname{Hom}_{p H D}\left(R \Gamma_{c}(\mathscr{X})(i)[n], \mathbb{K}\right)$.

We wish to point out that the constructions above are essentially consequences of the work done by Besser and Bannai, but it seems hard to prove the following results without the formalism of Godement resolutions that we develop in Section 3. Let $\mathscr{X}$ be a smooth scheme over $\mathscr{V}$. Then

(i) there is a cup product pairing

$$
H_{\mathrm{abs}}^{n}(\mathscr{X}, i) \otimes H_{\mathrm{abs}, c}^{m}(\mathscr{X}, j) \rightarrow H_{\mathrm{abs}, c}^{n+m}(\mathscr{X}, i+j)
$$

induced by the natural pairings defined on the cohomology of the generic and the special fiber (see Corollary 5.4.4),

(ii) there is a Poincaré duality isomorphism (see Proposition 5.4.5) and

(iii) there is a Gysin map; that is, given a proper morphism $f: \mathscr{X} \rightarrow \mathscr{Y}$ of smooth algebraic $\mathscr{V}$-schemes of relative dimension $d$ and $e$, respectively, then there is a canonical map

$$
f_{*}: H_{\mathrm{abs}}^{n}(\mathscr{X}, i) \rightarrow H_{\mathrm{abs}}^{n+2 c}(\mathscr{Y}, i+c),
$$

where $c=e-d$ (see Corollary 5.4.7).

Notation. In this paper, $R$ is a complete discrete valuation ring with fraction field $K$ and residue field $k$ with $k$ perfect. We assume $\operatorname{char}(K)=0$ and $\operatorname{char}(k)=p>0$.

The ring of Witt vectors of $k$ is denoted by $R_{0}$, and $K_{0}$ is its field of fractions. The Frobenius of $K_{0}$ is denoted by $\sigma$. The category of bounded complexes of $K$-vector spaces is denoted by $C^{b}(K)$.

If $V$ is a $K$-vector space, then $V^{\vee}$ is the dual vector space.

We use $X, Y, \ldots$ for schemes over $k$ or $K ; \mathscr{X}, \mathscr{Y}, \ldots$ for $K$-analytic spaces; $\mathrm{P}, \mathrm{Q}$ for formal $K$-schemes; $\mathrm{P}_{K}, \mathrm{Q}_{K}$ for the associated Raynaud fibers and $\mathscr{X}$, $\mathscr{Y}$ for (algebraic) $\mathscr{V}$-schemes, where $\mathscr{V}=\operatorname{Spec}(R)$. Finally, $\widehat{\mathscr{X}}$ denotes the $p$-adic completion of a $\mathscr{V}$-scheme $\mathscr{X}$.

\section{Cycle classes}

1.1. Higher Chow groups. Following Bloch [1986], we recall the definition of the higher Chow groups $C H^{*}(X / K, *)$ of a $K$-scheme $X$. For any $n \geq 0$, let 
$\Delta^{n}:=\operatorname{Spec}\left(\mathbb{Z}\left[t_{0}, \ldots, t_{n}\right] /\left(\sum_{i} t_{i}-1\right)\right)$ with face maps $\partial_{i}(n): \Delta^{n} \rightarrow \Delta^{n+1}$, which in coordinates are given by $\partial_{i}(n)\left(t_{0}, \ldots, t_{n}\right):=\left(t_{0}, \ldots, t_{i-1}, 0, t_{i+1}, \ldots, t_{n}\right)$. Let $X$ be a smooth $K$-scheme of relative dimension $d$ (this hypothesis is not necessary, but we deal only with smooth schemes). Let $z^{q}(X / K, 0)$ be the free abelian group generated by the irreducible and closed subschemes of $X$ of codimension $q$. Let $z^{q}(X / K, n)$ denote the free abelian group generated by the closed subschemes $W \subset \Delta_{X}^{n}:=X \times \Delta^{n}$ such that $W \in z^{q}\left(\Delta_{X}^{n} / K, 0\right)$ and meets all faces properly; that is, if $F \subset \Delta_{X}^{n}$ is a face of codimension $c$, then the codimension of each irreducible component of the intersection $F \cap W$ is greater than or equal to $c+q$ on $\Delta_{X}^{n}$.

Using the differential $\sum_{i}(-1)^{i} \partial_{i}^{*}(n): z^{q}(X / K, n+1) \rightarrow z^{q}(X / K, n)$, one obtains a complex of abelian groups $z^{q}(X / K, *)$. We set $\Gamma_{X}^{i}(q):=z^{q}(X / K, 2 q-i)$ and

$$
C H^{q}(X / K, 2 q-i):=H^{i}\left(\Gamma_{X}(q)\right) .
$$

These groups are in fact isomorphic to the Voevodsky-Suslin motivic cohomology $H_{m o t}^{i}(X / K, q)$ of the generic fiber $X$ [Mazza et al. 2006, Theorem 19.1].

1.2. Relative cycles. Let $\mathscr{X}$ be an algebraic and flat $\mathscr{V}$-scheme. By the theory of relative cycles [Suslin and Voevodsky 2000], one can define the group $z^{q}(\mathscr{X} / \mathscr{V}, 0)$ to be the free abelian group generated by universally integral relative cycles of codimension $q$ (we can use the codimension because $\mathscr{X}$ is assumed to be equidimensional over $\mathscr{V})$. By [Ivorra 2005, Part I, Lemma 1.2.6], $z^{q}(\mathscr{X} / \mathscr{V}, 0)$ is the free abelian group generated by the closed subschemes $\mathscr{W} \subset \mathscr{X}$ that are integral, of codimension $q$ and flat over $\mathscr{V}$. Then we can define the group $z^{q}(\mathscr{L} / \mathscr{V}, n)$ as the free abelian group generated by the integral and flat $\mathscr{V}$-schemes $\mathscr{W} \in z^{q}\left(\Delta_{\mathscr{L}}^{n}, 0\right)$ meeting all faces properly and such that $\partial_{i}(n-1)^{*} \mathcal{W}$ is flat over $\mathscr{V}$ for all $i$. Thus, we can form a complex $z^{q}(\mathscr{X} / \mathscr{V}, *)$ with the same boundary maps as $z^{q}(X / K, *)$.

Definition 1.2.1. With the notation above, we define the higher Chow groups of $\mathscr{X}$ over $\mathscr{V}$ to be

$$
C H^{q}(\mathscr{X} / \mathscr{V}, 2 q-i):=H^{i}\left(\Gamma_{X}(q)\right),
$$

where $\Gamma_{\mathscr{L} / \mathscr{V}}^{i}(q):=z^{q}(\mathscr{X} / \mathscr{V}, 2 q-i)$.

Remark 1.2.2. (i) Recall that by Lemma 5.1.1, any closed and flat subscheme of $\mathscr{X}$ is completely determined by its generic fiber. Then $z^{q}(\mathscr{X} / \mathscr{V}, *)$ is a subcomplex of $z^{q}\left(\mathscr{X}_{K} / K, *\right)$ inducing a canonical map in (co)homology

$$
\gamma: C H^{q}(\mathscr{X} / \mathscr{V}, 2 q-i):=H^{i}\left(\Gamma_{\mathscr{L}} / \mathcal{V}(q)\right) \rightarrow C H^{q}\left(\mathscr{X}_{K} / K, 2 q-i\right) .
$$

(ii) It follows easily that, for $i=2 q$, the map $\gamma: C H^{q}(\mathscr{X} / \mathscr{V}, 0) \rightarrow C H^{q}\left(\mathscr{X}_{K} / K, 0\right)$ is surjective by the snake lemma. In the general case, we don't know whether or not this map is injective or surjective. 
1.3. De Rham and rigid fundamental/cycle classes. In the following, we refer to [Hartshorne 1975; Baldassarri et al. 2004; Petrequin 2003] for the definitions and the properties of the (algebraic) de Rham and the rigid cohomology theory. Let $W$ be an integral scheme of dimension $r$ over $K$ (resp. over $k$ ). Then we can associate to $W$ its de Rham (resp. rigid) fundamental class, which is an element of the dual of the top de Rham (resp. rigid) cohomology with compact support

$$
[W]_{\mathrm{dR}}=\operatorname{tr}_{\mathrm{dR}} \in H_{\mathrm{dR}, c}^{2 r}(W)^{\vee} \quad\left(\text { resp. }[W]_{\text {rig }}=\operatorname{tr}_{\text {rig }} \in H_{\text {rig }, c}^{2 r}(W)^{\vee}\right) .
$$

For the de Rham case, this class is first defined in [Hartshorne 1975, Section 7] as an element of the de Rham homology; by Poincaré duality [Baldassarri et al. 2004, Theorem 3.4], it corresponds to the trace map. The rigid case is treated in [Petrequin 2003, Section 2.1, Section 6].

Now let $X$ be a $K$-scheme (resp. $k$-scheme) of dimension $d$ and $w=\sum_{i} n_{i} W_{i} \in$ $z^{q}(X / K, 0)$ (resp. $\left.\in z^{q}(X / k, 0)\right)$ be a dimension- $r$ cycle on $X$. The cohomology with compact support is functorial with respect to proper maps; hence, there is a canonical map

$$
f: \bigoplus_{i} H_{?, c}^{2 r}\left(W_{i}\right)^{\vee} \rightarrow H_{?, c}^{2 r}(|w|)^{\vee},
$$

where $|w|=\bigcup_{i} W_{i}$ is the support of $w$ and ? is dR or rig according to the choice of the base field. With the notation above, we define the de Rham (resp. rigid) cycle class of $w$ as

$$
[w]_{?}:=f\left(\sum_{i}\left[W_{i}\right]_{?}\right) \in H_{?, c}^{2 r}(|w|)^{\vee}, \quad \text { where } ?=\mathrm{dR}, \text { rig } .
$$

Again by functoriality, this defines an element of $H_{?, c}^{2 r}(X)^{\vee}$.

1.4. Compatibility. Let $\mathscr{X}$ be a flat $\mathscr{V}$-scheme, and let $w \in z^{q}(\mathscr{X} / \mathscr{V}, 0)$ be a relative cycle. We can write $w=\sum_{i} n_{i} \mathcal{W}_{i}$, where $\mathscr{W}_{i}$ is an integral flat $\mathscr{V}$-scheme closed in $\mathscr{X}$ and of codimension $q$. Then $w$ defines a cycle $w_{K} \in z^{q}\left(\mathscr{X}_{K} / K, 0\right)$ (resp. $\left.w_{k} \in z^{q}\left(\mathscr{X}_{k} / k, 0\right)\right)$; on the generic fiber, we get simply $w_{K}=\sum_{i} n_{i}\left(\mathscr{W}_{i}\right)_{K}$. However, on the special fiber, we must write the irreducible decomposition of each $\left(W_{i}\right)_{k}^{\text {red }}$, say $W_{i, 1} \cup \cdots \cup W_{i, r_{i}}$, and then consider the multiplicities, that is,

$$
w_{k}=\sum_{i}\left(n_{i} \sum_{j} m_{i, j} W_{i, j}\right),
$$

where $m_{i, j}:=$ length $\left(\mathcal{O}_{\mathscr{W}_{k}, W_{i, j}}\right)$.

We can consider the de Rham and the rigid cycle classes of $w$, that is,

$$
\left[w_{K}\right]_{\mathrm{dR}} \in H_{\mathrm{dR}, c}^{2 r}\left(\left|w_{K}\right|\right)^{\vee} \quad \text { and } \quad\left[w_{k}\right]_{\mathrm{rig}} \in H_{\mathrm{rig}, c}^{2 r}\left(\left|w_{k}\right|\right)^{\vee} .
$$


The rigid homology groups $H_{2 r}^{\text {rig }}\left(\left|w_{k}\right|\right)$ are defined as the dual of $H_{\text {rig, } c}^{2 r}\left(\left|w_{k}\right|\right)$; see [Petrequin 2003, Section 2]. We will prove that these cycle classes are compatible under (co)specialization and induce a well-defined syntomic cohomology class.

Theorem 1.4.1. Let $\mathscr{X}$ be a flat $\mathscr{V}$-scheme of relative dimension $d$, and let $w \in$ $z^{q}(\mathscr{X} / \mathcal{V}, 0)$ be a relative cycle of codimension $q$ (and relative dimension $r:=d-q$ ). Then $\operatorname{cosp}\left(\left[w_{k}\right]_{\mathrm{rig}}\right)=\left[w_{K}\right]_{\mathrm{dR}}$.

Proof. First of all, consider the basic case: $w=\mathscr{W}$ is an integral closed subscheme of $\mathscr{X}$ smooth over $\mathscr{V}$. By [Baldassarri et al. 2004, proof of Theorem 6.9], we have a commutative diagram

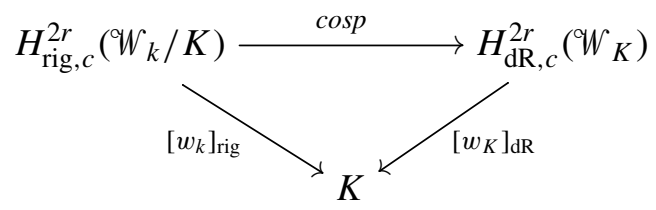

where the rigid (resp. de Rham) trace map $\left[w_{k}\right]_{\text {rig }}\left(\right.$ resp. $\left.\left[w_{K}\right]_{\mathrm{dR}}\right)$ is an isomorphism of $K$-vector spaces.

Given a general relative cycle $w$, we can reduce to the basic case by arguing as follows. First by linearity and the functoriality of the specialization map, we can restrict to the case $w=\mathscr{W}$ with $\mathscr{W}$ integral. Then the generic fiber $\mathscr{W}_{K}$ is integral, and by [Grothendieck 1967, Proposition 17.15.12], there exists a closed $K$ subscheme $T$ such that $\mathcal{W}_{K} \backslash T$ is smooth over $K$. Let $\mathscr{T}$ be the flat extension of $T$ (see Lemma 5.1.1); then $\mathscr{T}$ is of codimension at least 1 in $\mathscr{W}$, and the complement $\mathscr{W} \backslash \mathcal{T}$ is a flat $\mathscr{V}$-scheme of relative dimension $r$. Consider the long exact sequence

$$
\cdots H_{\mathrm{dR}, c}^{2 r-1}(T) \rightarrow H_{\mathrm{dR}, c}^{2 r}\left(\mathscr{W}_{K} \backslash \mathscr{T}_{K}\right) \rightarrow H_{\mathrm{dR}, c}^{2 r}\left(\mathscr{W}_{K}\right) \rightarrow H_{\mathrm{dR}, c}^{2 r}(T) \cdots
$$

Note that here the first and last terms vanish for dimensional reasons. The same happens (mutatis mutandis) for the rigid cohomology of the special fiber.

Hence, from now on, we can assume that $W$ is integral and that its generic fiber $\mathcal{W}_{K}$ is smooth. In this setting, we apply the reduced fiber theorem for schemes [Bosch et al. 1995, Theorem 2.1]; that is, there exist a finite field extension $K^{\prime} / K$ and a finite morphism $f: \mathscr{Y} \rightarrow \mathscr{W} \times \mathscr{V} \mathscr{V}^{\prime}$ of $\mathscr{V}^{\prime}$-schemes such that

(i) $f_{K^{\prime}}: \mathscr{Y}_{K^{\prime}} \rightarrow \mathscr{W}_{K^{\prime}}$ is an isomorphism and

(ii) $\mathcal{Y} / \mathscr{V}^{\prime}$ is flat and has reduced geometric fibers.

Recall that the cospecialization map commutes with finite field extension, and the same holds for both the rigid and de Rham trace maps. By [Petrequin 2003, proof of Proposition 6.4], the rigid fundamental/cycle class is preserved by finite morphisms, that is,

$$
\left[\mathscr{Y}_{k^{\prime}}\right]_{\text {rig }} \circ f_{k^{\prime}}^{*}=\left[\mathscr{W}_{k^{\prime}}\right]_{\text {rig }} \quad \text { where } f_{k^{\prime}}^{*}: H_{\text {rig,c }}^{2 r}\left(\mathscr{W}_{k^{\prime}}\right) \rightarrow H_{\text {rig, }, c}^{2 r}\left(\mathscr{Y}_{k^{\prime}}\right) .
$$


From the discussion above, there is no loss of generality in assuming that $W$ has reduced geometric fibers and smooth generic fiber $\mathcal{W}_{K}$. Now let $S$ be the singular locus of the special fiber $\mathscr{W}_{k}$. Again by [Grothendieck 1967, Proposition 17.15.12], the complement $\mathscr{W}_{k} \backslash S$ is an open and dense subscheme of $\mathscr{W}_{k}$, and it is smooth over $k$. The scheme $S$ has codimension at least 1 in $\mathcal{W}_{k}$; hence, $H_{\mathrm{rig}, c}^{2 r}\left(\mathcal{W}_{k} \backslash S\right) \rightarrow H_{\mathrm{rig}, c}^{2 r}\left(\mathscr{W}_{k}\right)$ is an isomorphism. From this, it follows that we can assume $\mathcal{W}$ to be smooth over $\mathscr{V}$, where we know that the claim is true.

1.5. The smooth case. From now on, assume $\mathscr{X}$ to be smooth over $\mathscr{V}$. By the compatibility of (co)specialization with Poincaré duality [Baldassarri et al. 2004, Theorem 6.9], the (de Rham or rigid) cycle class map defines an element $\eta_{\text {rig }}\left(w_{k}\right) \in$ $H_{\text {rig, }\left|w_{k}\right|}^{2 q}\left(\mathscr{X}_{k} / K\right)\left(\operatorname{resp} . \eta_{\mathrm{dR}}\left(w_{K}\right) \in H_{\mathrm{dR},\left|w_{K}\right|}^{2 q}\left(\mathscr{X}_{K}\right)\right)$ compatible with respect to the specialization morphism

$$
s p\left(\eta_{\mathrm{dR}}\left(w_{K}\right)\right)=\eta_{\text {rig }}\left(w_{k}\right) .
$$

Before stating the next corollary, we need to introduce some further notation. Let $X$ be a smooth scheme over $K$ (resp. over $k$ ); then the de Rham (resp. rigid) cycle class map factors through the Chow groups, inducing a map

$$
\eta_{\mathrm{dR}}: C H^{q}(X / K) \rightarrow H_{\mathrm{dR}}^{2 q}(X) \quad\left(\text { resp. } \eta_{\text {rig }}: C H^{q}(X / k) \rightarrow H_{\text {rig }}^{2 q}(X / K)\right),
$$

where, by abuse of notation, $\eta_{\mathrm{dR}}(W)$ (resp. $\eta_{\mathrm{rig}}(W)$ ) is viewed as an element of $H_{\mathrm{dR}}^{2 q}(X)\left(\right.$ resp. $H_{\text {rig }}^{2 q}(X / K)$ ) via the canonical map $H_{\mathrm{dR}, W}^{2 q}(X) \rightarrow H_{\mathrm{dR}}^{2 q}(X)$ (resp. $\left.H_{\text {rig, } W}^{2 q}(X / K) \rightarrow H_{\text {rig }}^{2 q}(X / K)\right)$ for any $W$ integral subscheme of codimension $q$ [Hartshorne 1975, Proposition 7.8.1, page 60; Petrequin 2003, Corollary 7.6].

Also, we recall that in [Berthelot et al. 1971, Exp. X, Appendix], a specialization map for the classical Chow ring is constructed:

$$
s p_{C H}: C H^{*}\left(\mathscr{L}_{K} / K\right) \otimes \mathbb{Q} \rightarrow C H^{*}\left(\mathscr{X}_{k} / k\right) \otimes \mathbb{Q} .
$$

Explicitly, the map is given as follows. Let $W \subset \mathscr{X}_{K}$ be an integral scheme of codimension $q$, and let $\mathscr{W}$ denote its Zariski closure in $\mathscr{X}$. Then the specialization of $s p_{C H}[W]$ is the class representing the subscheme $\mathcal{W}_{k}$.

Corollary 1.5.1. With the notation above, the diagram

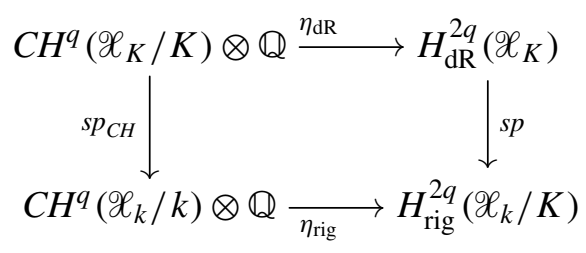

commutes (we tensored each term by $\mathbb{Q}$ to guarantee the existence of $s p_{C H}$ ). 
1.6. Syntomic cohomology. For any smooth $\mathscr{V}$-scheme $\mathscr{X}$, Besser [2000, Definition 6.1] defined the (rigid) syntomic cohomology groups $H_{\text {syn }}^{n}(\mathscr{X}, i)$. We will be rather sketchy on the definitions because we will give another construction later. For such a cohomology, there is a long exact sequence

$$
\begin{aligned}
\longrightarrow H_{\text {syn }}^{n}(\mathscr{X}, i) \longrightarrow H_{\text {rig }}^{n}\left(\mathscr{X}_{k} / K_{0}\right) \oplus F^{i} H_{\mathrm{dR}}^{n}\left(\mathscr{X}_{K}\right) \\
\stackrel{h}{\longrightarrow} H_{\text {rig }}^{n}\left(\mathscr{X}_{k} / K_{0}\right) \oplus H_{\text {rig }}^{n}\left(\mathscr{X}_{k} / K\right) \stackrel{+}{\longrightarrow}
\end{aligned}
$$

where $h\left(x_{0}, x_{\mathrm{dR}}\right)=\left(\phi\left(x_{0}^{\sigma}\right)-p^{i} x_{0}, x_{0} \otimes 1_{K}-s p\left(x_{\mathrm{dR}}\right)\right)$.

Roughly, these groups are defined as $H_{\text {syn }}^{n}(\mathscr{X}, i):=H^{n}\left(\mathbb{R} \Gamma_{B e s}(\mathscr{X}, i)\right)$, where $\mathbb{R} \Gamma_{\text {Bes }}(\mathscr{Q}, i)$

$$
:=\operatorname{Cone}\left(\mathbb{R} \Gamma_{\text {rig }}\left(\mathscr{X} / K_{0}\right) \oplus F i l^{i} \mathbb{R} \Gamma_{\mathrm{dR}}(\mathscr{X}) \rightarrow \mathbb{R} \Gamma_{\text {rig }}\left(\mathscr{X} / K_{0}\right) \oplus \mathbb{R} \Gamma_{\text {rig }}(\mathscr{X} / K)\right)[-1]
$$

is a complex of abelian groups functorial in $\mathscr{X}$ and such that

$$
H^{n}\left(\mathbb{R} \Gamma_{\text {rig }}\left(\mathscr{X} / K_{0}\right)\right)=H_{\text {rig }}^{n}\left(\mathscr{X}_{k} / K_{0}\right) \text { and } H^{n}\left(F i l^{i} \mathbb{R} \Gamma_{\mathrm{dR}}(\mathscr{X})\right)=F^{i} H_{\mathrm{dR}}^{n}\left(\mathscr{X}_{K}\right) .
$$

The functoriality of $\mathbb{R} \Gamma_{\mathrm{Bes}}(\cdot, i)$ allows us to give the following definition:

Definition 1.6.1. Let $\mathscr{X}$ be a smooth $\mathscr{V}$-scheme. Let $\mathscr{L} \subset \mathscr{X}$ be a closed subscheme of $\mathscr{X}$. We define the syntomic complex with support in $\mathscr{E}$ using the complexes defined by Besser as

$$
\mathbb{R} \Gamma_{\text {Bes }, \mathscr{L}}(\mathscr{X}, i):=\operatorname{Cone}\left(\mathbb{R} \Gamma_{\text {Bes }}(\mathscr{X}, i) \rightarrow \mathbb{R} \Gamma_{\text {Bes }}(\mathscr{X} \backslash \mathscr{L}, i)\right)[-1] .
$$

This is a complex of abelian groups functorial with respect to cartesian squares. This fact will be used in the proof of Proposition 1.6.6. The cohomology of this complex is the syntomic cohomology with support in $\mathscr{L}$ denoted by

$$
H_{\mathrm{syn}, \mathscr{L}}^{n}(\mathscr{X}, i):=H^{n}\left(\mathbb{R} \Gamma_{\mathrm{Bes}, \mathscr{L}}(\mathscr{X}, i)\right)
$$

so that we get a long exact sequence

$$
\cdots H_{\mathrm{syn}, \mathscr{L}}^{n}(\mathscr{X}, i) \rightarrow H_{\mathrm{syn}}^{n}(\mathscr{X}, i) \rightarrow H_{\mathrm{syn}}^{n}(\mathscr{X} \backslash \mathscr{L}, i) \rightarrow \cdots .
$$

Proposition 1.6.2 (syntomic cycle class). Let $\mathscr{X}$ be a smooth $\mathscr{V}$-scheme, and let $w \in z^{q}(\mathscr{X} / \mathcal{V}, 0)$ be a relative cycle of codimension $q$. Then the canonical mapping

$$
\psi: H_{\mathrm{syn},|w|}^{2 q}(\mathscr{X}, q) \rightarrow H_{\mathrm{rig},\left|w_{k}\right|}^{2 q}\left(\mathscr{C}_{k} / K_{0}\right) \oplus F^{q} H_{\mathrm{dR},\left|w_{K}\right|}^{2 q}\left(\mathscr{X}_{K}\right)
$$

is injective, and there exists a unique element $\eta_{\mathrm{syn}}(w) \in H_{\mathrm{syn},|w|}^{2 q}(\mathscr{X}, q)$ such that $\psi\left(\eta_{\mathrm{syn}}(w)\right)=\left(\eta_{\text {rig }}\left(w_{k}\right), \eta_{\mathrm{dR}}\left(w_{K}\right)\right)$. 
Proof. By the definition of syntomic cohomology with support, there is a long exact sequence similar to (2):

$$
\begin{aligned}
\rightarrow & H_{\text {rig, },\left|w_{k}\right|}^{2 q-1}\left(\mathscr{X}_{k} / K_{0}\right) \oplus H_{\mathrm{rig},\left|w_{k}\right|}^{2 q-1}\left(\mathscr{X}_{k} / K\right) \rightarrow H_{\mathrm{syn},|w|}^{2 q}(X, q) \rightarrow \\
& H_{\mathrm{rig},\left|w_{k}\right|}^{2 q}\left(\mathscr{X}_{k} / K_{0}\right) \oplus F^{q} H_{\mathrm{dR},\left|w_{K}\right|}^{2 q}\left(\mathscr{X}_{K}\right) \rightarrow H_{\mathrm{rig},\left|w_{k}\right|}^{2 q}\left(\mathscr{X}_{k} / K_{0}\right) \oplus H_{\mathrm{rig},\left|w_{k}\right|}^{2 q}\left(\mathscr{X}_{k} / K\right) \rightarrow .
\end{aligned}
$$

The last term on the left vanishes because of weak purity in rigid cohomology [Berthelot 1997b, Corollary 5.7]. It follows that $H_{\mathrm{syn},|w|}^{2 q}(\mathscr{X}, q)$ consists of the pairs $(x, y) \in H_{\text {rig, }\left|w_{k}\right|}^{2 q}\left(\mathscr{X}_{k} / K_{0}\right) \oplus F^{q} H_{\mathrm{dR},\left|w_{K}\right|}^{2 q}\left(\mathscr{X}_{K}\right)$ such that $\phi\left(x^{\sigma}\right)=p^{q} x$ and $s p(y)=x \otimes 1_{K}$. By Hodge theory, we have $F^{q} H_{\mathrm{dR},\left|w_{K}\right|}^{2 q}\left(\mathscr{L}_{K}\right)=H_{\mathrm{dR},\left|w_{K}\right|}^{2 q}\left(\mathscr{X}_{K}\right)$. Moreover, the Frobenius acts on $\left[w_{k}\right]_{\text {rig }}$ as multiplication by $p^{q}$ [Petrequin 2003, Proposition 7.13]. Hence, in view of (1), we can easily conclude the proof.

Lemma 1.6.3 (functoriality). Let $f: \mathscr{X}^{\prime} \rightarrow \mathscr{X}$ be a closed immersion of smooth $\mathscr{V}$-schemes, and let $w \in z^{q}(\mathscr{X} / \mathscr{V}, 0)$ be a relative cycle of codimension $q$. Assume that the preimage $f^{-1} w$ lies in $z^{q}\left(\mathscr{X}^{\prime} / \mathscr{V}, 0\right)$; then $f^{*} \eta_{\mathrm{syn}}(w)=\eta_{\mathrm{syn}}\left(f^{-1} w\right)$.

Proof. It is not restrictive to assume that $w=\mathscr{W}$ is an integral subscheme of $\mathscr{L}$ flat over $\mathscr{V}$.

We first show that it is sufficient to prove that $f^{*} \eta_{\mathrm{dR}}\left(w_{K}\right)=\eta_{\mathrm{dR}}\left(f^{-1} w_{K}\right)$. In fact, the syntomic cycle class can be viewed as an element $\left(\eta_{\mathrm{rig}}\left(w_{k}\right), \eta_{\mathrm{dR}}\left(w_{K}\right)\right)$ in the direct sum of rigid and de Rham cohomology. Then note that $\operatorname{sp}\left(\eta_{\mathrm{dR}}\left(w_{K}\right)\right)=$ $\eta_{\text {rig }}\left(w_{k}\right) \otimes 1_{K}$ and that the specialization map is functorial.

To prove that $f^{*} \eta_{\mathrm{dR}}\left(W_{K}\right)=\eta_{\mathrm{dR}}\left(f^{-1} W_{K}\right)$, we first reduce by excision to the case where $\mathscr{W}_{K}$ is smooth over $K$ (just remove from $\mathcal{W}_{K}, \mathscr{X}_{K}$ and $\mathscr{X}_{K}^{\prime}$ the singular points of $\mathcal{W}_{K}$ ). In the same way, we can further assume $f^{-1} \mathcal{W}_{K}$ to be smooth over $K$. Now we can use the same proof as [Petrequin 2003, Proposition 7.1] to conclude.

Lemma 1.6.4 (homotopy). The (rigid) syntomic cohomology is homotopy invariant:

$$
H_{\text {syn }}^{n}\left(\mathscr{X} \times \mathbb{A}_{\mathscr{V}}^{1}, q\right) \cong H_{\text {syn }}^{n}(\mathscr{X}, q) .
$$

Proof. Just consider the long exact sequences of syntomic cohomology, and note that the de Rham cohomology (of smooth schemes) is homotopy invariant by [Hartshorne 1975, Proposition 7.9.1]. The same holds for rigid cohomology, for instance, using the Künneth formula [Berthelot 1997a].

Lemma 1.6.5 (weak purity). Let $\mathscr{X}$ be a smooth $\mathscr{V}$-scheme. Let $\mathscr{E} \subset \mathscr{X}$ be a closed subscheme of $\mathscr{Q}$ of codimension $q$. Then

$$
H_{\mathrm{syn}, \mathscr{L}}^{n}(\mathscr{X}, i)=0 \text { for all } n<2 q .
$$


Proof. This follows directly from the long exact sequence of syntomic cohomology and the weak purity in de Rham and rigid cohomology [Hartshorne 1975, Section 7.2; Petrequin 2003, Section 1].

Proposition 1.6.6. Let $\mathscr{L}$ be a smooth $\mathscr{V}$-scheme. The syntomic cycle class map induces a group homomorphism $\mathrm{reg}_{\mathrm{syn}}: \mathrm{CH}^{i}(\mathscr{X} / \mathcal{V}, 2 i-n) \rightarrow H_{\mathrm{syn}}^{n}(\mathscr{X}, i)$.

Proof. The construction is analogous to that provided in [Bloch 1986]. Consider the cohomological double complex $\mathbb{R} \Gamma_{\mathrm{Bes}}\left(\Delta_{\mathscr{X}}^{-n}, q\right)^{m}$ nonzero for $m \geq 0$ and $n \leq 0$; the differential in $n$ is induced by $\partial_{i}^{(-n)}$ in the usual way. Similarly define the double complex

$$
\mathbb{R} \Gamma_{\text {Bes,supp }}\left(\Delta_{\mathscr{X}}^{-n}, q\right)^{m}:=\operatorname{colim}_{w \in z^{q}(\mathscr{X},-n)} \mathbb{R} \Gamma_{\text {Bes },|w|}\left(\Delta_{\mathscr{X}}^{-n}, q\right)^{m} .
$$

For technical reasons, we truncate these complexes (nontrivially):

$$
A_{?}^{n, m}=\tau_{n \geq-N} \mathbb{R} \Gamma_{\text {Bes,? }}\left(\Delta_{\mathscr{L}}^{-n}, q\right)^{m}, \quad \text { where } ?=\varnothing, \text { supp, }
$$

for $N$ even and $N \gg 0$.

Consider the spectral sequence

$$
E_{1}^{n, m}:=H^{m}\left(A^{\bullet, n}\right) \Rightarrow H^{n+m}\left(s A^{\bullet, *}\right),
$$

where $s$ denotes the associated simple complex of a double complex. By homotopy invariance (Lemma 1.6.4), $E_{1}^{n, m}:=H_{\mathrm{syn}}^{m}\left(\Delta_{\mathscr{X}}^{-n}, q\right)$ is isomorphic to $H_{\mathrm{syn}}^{m}(\mathscr{X}, q)$ for $-N \leq n \leq 0$ and $m \geq 0$; otherwise, it is 0 . Moreover, $d_{1}^{n, m}=0$ except for $n$ even, $-N \leq n<0$ and $m \geq 0$, in which case $d_{1}^{n, m}=\mathrm{id}$. This gives an isomorphism $H^{i}\left(s A^{n, m}\right) \cong H_{\text {syn }}^{i}(\mathscr{X}, q)$.

In the spectral sequence

$$
E_{1, \text { supp }}^{n, m}:=H^{m}\left(A_{\text {supp }}^{\bullet, n}\right) \Rightarrow H^{n+m}\left(s A_{\text {supp }}^{\bullet, *}\right),
$$

we have

$$
E_{1, \text { supp }}^{n, m}=\operatorname{colim}_{w \in z^{q}(X,-n)} H_{\mathrm{syn},|w|}^{m}\left(\Delta_{\mathscr{X}}^{-n}, q\right) \quad \text { for }-N \leq n \leq 0 \text { and } m \geq 0
$$

and $E_{1, \text { supp }}^{n, m}=0$ otherwise. Applying Lemma 1.6.3 to the face morphisms, it is easy to prove that the syntomic cycle class induces a natural map of complexes $\Gamma_{\mathscr{X} / \mathscr{V}}^{\bullet}(q) \rightarrow E_{1, \text { supp }}^{\bullet-2 q, 2 q}$, and hence, for all $i$, a map $C H^{q}(\mathscr{X} / \mathscr{V}, 2 q-i) \rightarrow E_{2, \text { supp }}^{i-2 q, 2 q}$. The groups $E_{r, \text { supp }}^{n, m}$ are zero for $m<2 q$ and $r \geq 1$ due to weak purity. Hence, there are natural maps $E_{2, \text { supp }}^{i-2 q, 2 q} \rightarrow E_{\infty, \text { supp }}^{i-2 q, 2 q} \rightarrow H^{i}\left(s A_{\text {supp }}^{\bullet, *}\right)$. By construction, there is a map $H^{i}\left(s A_{\text {supp }}^{\bullet, *}\right) \rightarrow H^{i}\left(s A^{\bullet, *}\right)$. Composing all these maps, we obtain the expected map $C H^{q}(\mathscr{X}, 2 q-i) \rightarrow H_{\text {syn }}^{i}(\mathscr{X}, q)$. 
Corollary 1.6.7. With the notation above, there is a commutative diagram

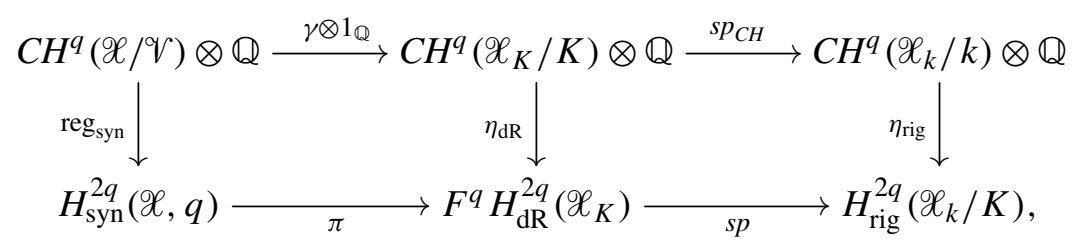

where $\pi$ is the composition

$$
H_{\mathrm{syn}}^{n}(\mathscr{X}, i) \longrightarrow H_{\mathrm{rig}}^{n}\left(\mathscr{X}_{k} / K_{0}\right) \oplus F^{i} H_{\mathrm{dR}}^{n}\left(\mathscr{X}_{K}\right) \stackrel{\mathrm{pr}_{2}}{\longrightarrow} F^{i} H_{\mathrm{dR}}^{n}\left(\mathscr{X}_{K}\right)
$$

and $\gamma$ is the map described in Remark 1.2.2.

Proof. Just note that in this case, reg $_{\text {syn }}$ is the map induced by the syntomic cycle class in the usual way.

Remark 1.6.8. Via Chern classes, Besser [2000, Theorem 7.5] obtained a regulator $c_{i}^{2 i-n}: K_{2 i-n}(X) \rightarrow H_{\text {syn }}^{n}(\mathscr{X}, i)$. At present, we cannot compare it with the regulator of Proposition 1.6.6. This is because we don't know how to relate $K$-theory with the higher Chow groups we have defined. Nevertheless, we expect that there exists a map $C H^{i}(\mathscr{X} / \mathscr{V}, 2 i-n)_{\mathbb{Q}} \rightarrow K_{2 i-n}(X)_{\mathbb{Q}}$. This issue will be treated in a future work.

\section{2. $p$-adic Hodge complexes}

Having defined a regulator map with values in the syntomic cohomology, it is tempting to check (some of) the Bloch-Ogus axioms for this theory. We address this problem by viewing the syntomic cohomology as an absolute cohomology theory.

Thus, in this section, we define a triangulated category of p-adic Hodge complexes similar to that of [Bannai 2002]. See also [Berlinson 1986; Huber 1995; Levine 1998, Chapter V, Section 2.3].

The syntomic cohomology will be computed by Hom groups in this category.

Definition 2.0.9 (see [Bannai 2002, Section 2]). Let $C_{\text {rig }}^{b}(K)$ be the category of pairs $\left(M^{\bullet}, \phi\right)$, where

(i) $M^{\bullet}=M_{0}^{\bullet} \otimes_{K_{0}} K$ and $M_{0}^{\bullet}$ is a complex in $C^{b}\left(K_{0}\right)$;

(ii) (Frobenius structure) if $\left(M_{0}^{\bullet}\right)^{\sigma}:=M_{0}^{\bullet} \otimes_{\sigma} K_{0}$, then $\phi:\left(M_{0}^{\bullet}\right)^{\sigma} \rightarrow M_{0}^{\bullet}$ is a $K_{0}$-linear morphism.

The morphisms in this category are morphisms in $C^{b}\left(K_{0}\right)$ compatible with respect to the Frobenius structure. In this way, we get an abelian category.

Let Filt ${ }_{K}$ be the category of $K$-vector spaces with a descending, exhaustive and separated filtration. We write $C_{\mathrm{dR}}^{b}(K)=C^{b}\left(\right.$ Filt $\left._{K}\right)$, and we write the objects of this category as pairs $\left(M^{\bullet}, F\right)$, where 
(i) $M^{\bullet}$ is a complex in $C^{b}(K)$ and

(ii) (Hodge filtration) $F$ is a (separated and exhaustive) filtration on $M^{\bullet}$.

Remark 2.0.10 (strictness). We review some technical facts about filtered categories. For a full discussion, see [Huber 1995, Sections 2 and 3].

The category Filt $_{K}$ (and also $C_{\mathrm{dR}}^{b}(K)$ ) is additive but not abelian. It is an exact category when one takes for short exact sequences those that are exact as sequences of $K$-vector spaces and are such that the morphisms are strict with respect to the filtrations; recall that a morphism $f:(M, F) \rightarrow(N, F)$ is strict if $f\left(F^{i} M\right)=F^{i} N \cap \operatorname{Im}(f)$.

An object $\left(M^{\bullet}, F\right) \in C_{\mathrm{dR}}^{b}(K)$ is a strict complex if its differentials are strict as morphism of filtered vector spaces. Strict complexes can be characterized also by the fact that the canonical spectral sequence $H^{q}\left(F^{p}\right) \Rightarrow H^{p+q}\left(M^{\bullet}\right)$ degenerates at $E_{1}$.

One can define canonical truncation functors on $C_{\mathrm{dR}}^{b}(K)$ : For $M^{\bullet} \in C_{\mathrm{dR}}^{b}(K)$, let $\tau_{\leq n}\left(M^{\bullet}, F\right)^{i}:=\left\{\begin{array}{ll}M^{i} & \text { if } i<n, \\ \operatorname{Ker}\left(d^{n}\right) & \text { if } i=n, \\ 0 & \text { if } i>n,\end{array} \quad \tau_{\geq n}\left(M^{\bullet}, F\right)^{i}:= \begin{cases}0 & \text { if } i<n-1, \\ \operatorname{Coim}\left(d^{n}\right) & \text { if } i=n-1, \\ M^{i} & \text { if } i \geq n .\end{cases}\right.$

It is important to note that the naive cohomology object $\tau_{\leq n} \tau_{\geq n}\left(M^{\bullet}, F\right)$ of a strict complex $\left(M^{\bullet}, F\right)$ agrees with the cohomology $H^{n}\left(M^{\bullet}\right)$ of the complex of $K$-vector spaces underlying $\left(M^{\bullet}, F\right)$ [Huber 1995, Proposition 2.1.3 and Section 3].

Definition 2.0.11 (see [Bannai 2002, Definition 2.2]). From the discussion above, there is an exact functor $\Phi_{\text {rig }}: C_{\text {rig }}^{b}(K) \rightarrow C^{b}(K)\left(\right.$ resp. $\left.\Phi_{\mathrm{dR}}: C_{\mathrm{dR}}^{b}(K) \rightarrow C^{b}(K)\right)$ induced by $\left(M^{\bullet}, \phi\right) \mapsto M^{\bullet}$ (resp. $\left.\left(M^{\bullet}, F\right) \mapsto M^{\bullet}\right)$. We define the category $p H C$ of $p$-adic Hodge complexes whose objects are systems $M=\left(M_{\mathrm{rig}}^{\bullet}, M_{\mathrm{dR}}^{\bullet}, M_{K}^{\bullet}, c, s\right)$, where

(i) $\left(M_{\text {rig }}^{\bullet}, \phi\right)$ is an object of $C_{\text {rig }}^{b}(K)$ and $H^{*}\left(M_{\text {rig }}^{\bullet}\right)$ is finitely generated over $K$,

(ii) $\left(M_{\mathrm{dR}}^{\bullet}, F\right)$ is an object of $C_{\mathrm{dR}}^{b}(K)$ and $H^{*}\left(M_{\mathrm{dR}}^{\bullet}\right)$ is finitely generated over $K$ and (iii) $M_{K}^{\bullet}$ is an object of $C^{b}(K)$ and $c: M_{\text {rig }}^{\bullet} \rightarrow M_{K}^{\bullet}\left(\right.$ resp. $s: M_{\mathrm{dR}}^{\bullet} \rightarrow M_{K}^{\bullet}$ ) is a morphism in $C^{b}(K)$. Hence, $c$ and $s$ give a diagram in $C_{K}^{b}$

$$
M_{\text {rig }}^{\bullet} \stackrel{c}{\rightarrow} M_{K}^{\bullet} \stackrel{s}{\leftarrow} M_{\mathrm{dR}}^{\bullet}
$$

A morphism in $p H C$ is given by a system $f:=\left(f_{\text {rig }}, f_{\mathrm{dR}}, f_{K}\right)$, where $f_{?}: M_{?}^{\bullet} \rightarrow$ $N_{?}^{\bullet}$ is a morphism in $C_{\text {rig }}^{b}(K), C_{\mathrm{dR}}^{b}(K)$ or $C^{b}(K)$ for ? = rig, $\mathrm{dR}$ or $K$, respectively, and such that they are compatible with respect to the diagram in (iii) above. 
2.1. Derived version. A homotopy in $p H C$ is a system of homotopies $h_{i}$ compatible with the comparison maps $c$ and $s$. We define the category $p H K$ to be the category $\mathrm{pHC}$ modulo morphisms homotopic to 0 . We say that a morphism $f=\left(f_{\mathrm{rig}}, f_{\mathrm{dR}}, f_{K}\right)$ in $p H C$ (or $\left.p H K\right)$ is a quasi-isomorphism if $f_{\text {? }}$ is a quasiisomorphism for ? = rig (or $K$ ) and $f_{\mathrm{dR}}$ is a filtered quasi-isomorphism, that is, $\mathrm{gr}_{F}\left(f_{\mathrm{dR}}\right)$ is a quasi-isomorphism. Finally, we say that $M \in p H C$ (or $p H K$ ) is acyclic if $M_{?}=0$ is acyclic for any ? = rig, $\mathrm{dR}, K$.

Lemma 2.1.1. (i) The category $\mathrm{pHK}$ is a triangulated category.

(ii) The localization of $\mathrm{pHK}$ with respect to the class of quasi-isomorphisms exists. This category, denoted $p H D$, is a triangulated category.

(iii) On the category $p H D$, it is possible to define a nondegenerate $t$-structure (resp. truncation functors) compatible with the standard $t$-structure (resp. truncation functors) defined on $C^{b}(K), C_{\mathrm{rig}}^{b}(K)$ and $C_{\mathrm{dR}}^{b}(K)$.

Proof. The proof is the same as [Bannai 2002, Proposition 2.6]. See also [Huber 1995, Section 2] for a survey on how to derive exact categories.

Remark 2.1.2. In the terminology of Huber [1995, Section 4], the category $p H C$ is the glued exact category of $C_{\mathrm{rig}}^{b}$ and $C_{\mathrm{dR}}^{b}$ via $C_{K}^{b}$. The foregoing definition is inspired by Bannai [2002], who constructs a rigid glued exact category $C_{M F}^{b}$, that is, the comparison maps are all quasi-isomorphisms. For our purposes, we cannot impose this strong assumption. This is motivated by the fact that the (co)specialization is not an isomorphism for a general smooth $\mathscr{V}$-scheme.

2.2. Derived Hom. Let $M^{\bullet}$ and $M^{\prime \bullet}$ be two objects of $p H C$. Consider the diagram $\mathscr{H}\left(M^{\bullet}, M^{\prime \bullet}\right)$ (of complexes of abelian groups)

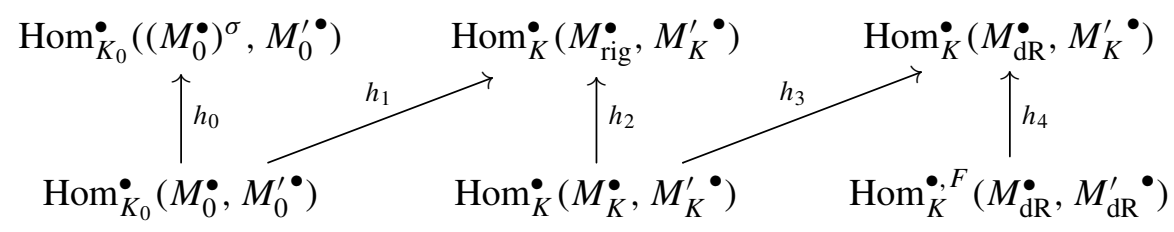

where $h_{0}\left(x_{0}\right)=x_{0} \circ \phi-\phi^{\prime} \circ x_{0}^{\sigma} ; h_{1}\left(x_{0}\right)=c^{\prime} \circ\left(x_{0} \otimes \operatorname{id}_{K}\right) ; h_{2}\left(x_{K}\right)=x_{K} \circ c$; $h_{3}\left(x_{K}\right)=x_{K} \circ s$ and $h_{4}\left(x_{\mathrm{dR}}\right)=s^{\prime} \circ x_{\mathrm{dR}} ; \operatorname{Hom}_{K}^{\bullet, F}\left(M_{\mathrm{dR}}^{\bullet}, M_{\mathrm{dR}}^{\prime} \bullet\right)$ is the complex of morphisms compatible with respect to the filtrations. Then define the two complexes of abelian groups $\Gamma_{0}\left(M^{\bullet}, M^{\prime \bullet}\right):=$ (direct sum of the bottom row) and $\Gamma_{1}\left(M^{\bullet}, M^{\prime \bullet}\right):=$ (direct sum of the top row). Finally, consider the cone

$$
\Gamma\left(M^{\bullet}, M^{\prime \bullet}\right):=\operatorname{Cone}\left(\psi_{M^{\bullet}, M^{\prime \bullet}}: \Gamma_{0}\left(M^{\bullet}, M^{\prime \bullet}\right) \rightarrow \Gamma_{1}\left(M^{\bullet}, M^{\prime \bullet}\right)\right)[-1],
$$

where

$$
\psi_{M} \cdot M^{\prime} \cdot\left(x_{0}, x_{K}, x_{\mathrm{dR}}\right) \mapsto\left(-h_{0}\left(x_{0}\right), h_{1}\left(x_{0}\right)-h_{2}\left(x_{K}\right), h_{3}\left(x_{K}\right)-h_{4}\left(x_{\mathrm{dR}}\right)\right) .
$$


Remark 2.2.1. (i) Let $\mathbb{K}(-n)$ be the Tate twisted $p$-adic Hodge complex: i.e., $\mathbb{K}(-n)_{\text {rig }}$ (resp. $\left.\mathbb{K}(-n)_{\mathrm{dR}}, \mathbb{K}(-n)\right)$ is equal to $K$ concentrated in degree 0 ; the Frobenius is $\phi(\lambda):=p^{n} \sigma(\lambda)$; the filtration is $F^{i}=K$ for $i \leq n$ and 0 otherwise.

(ii) Given two $p$-adic Hodge complexes $M^{\bullet}$ and $M^{\prime \bullet}$, we define their tensor product $M^{\bullet} \otimes M^{\prime \bullet}$ component-wise, that is, $\left(M_{\mathrm{rig}}^{\bullet} \otimes M_{\mathrm{rig}}^{\prime} \cdot M_{\mathrm{dR}}^{\bullet} \otimes M_{\mathrm{dR}}^{\prime} \cdot M_{K}^{\bullet} \otimes M_{K}^{\prime} \bullet\right.$, $\left.c \otimes c^{\prime}, s \otimes s^{\prime}\right)$. The complex $M^{\bullet} \otimes \mathbb{K}(n)$ is denoted by $M^{\bullet}(n)$.

(iii) The complex $\Gamma\left(\mathbb{K}, M^{\bullet}(n)\right)$ is quasi-isomorphic to

$$
\begin{gathered}
\operatorname{Cone}\left(M_{0}^{\bullet} \oplus F^{n} M_{\mathrm{dR}}^{\bullet} \stackrel{\eta}{\rightarrow} M_{0}^{\bullet} \oplus M_{K}^{\bullet}\right)[-1], \\
\eta\left(x_{0}, x_{\mathrm{dR}}\right)=\left(p^{-n} \phi\left(x_{0}^{\sigma}\right)-x_{0}, c\left(x_{0} \otimes \mathrm{id}_{K}\right)-s\left(x_{\mathrm{dR}}\right)\right),
\end{gathered}
$$

where $x_{0} \in M_{0}^{\bullet}$ and $x_{\mathrm{dR}} \in F^{n} M_{\mathrm{dR}}^{\bullet}$.

If $c$ is a quasi-isomorphism, letting $s p$ denote the composition of

$$
H^{q}\left(F^{n} M_{\mathrm{dR}}^{\bullet} \stackrel{s^{*}}{\rightarrow} H^{q}\left(M_{K}^{\bullet}\right) \stackrel{c^{*}}{\cong} H^{q}\left(M_{\mathrm{rig}}^{\bullet}\right),\right.
$$

we obtain a long exact sequence

$\rightarrow H^{q}\left(\Gamma\left(\mathbb{K}, M^{\bullet}(n)\right)\right) \rightarrow H^{q}\left(M_{0}^{\bullet}\right) \oplus H^{q}\left(F^{n} M_{\mathrm{dR}}^{\bullet} \stackrel{\eta^{\prime}}{\rightarrow} H^{q}\left(M_{0}^{\bullet}\right) \oplus H^{q}\left(M_{\mathrm{rig}}^{\bullet}\right) \rightarrow\right.$, where $\eta^{\prime}\left(x_{0}, x_{\mathrm{dR}}\right)=\left(p^{-n} \phi\left(x_{0}^{\sigma}\right)-x_{0}, x_{0} \otimes 1_{K}-s p\left(x_{\mathrm{dR}}\right)\right)$.

If $s$ is a quasi-isomorphism, letting cosp denote the composition of

$$
H^{q}\left(M_{\mathrm{rig}}^{\bullet}\right) \stackrel{c^{*}}{\rightarrow} H^{q}\left(M_{K}^{\bullet}\right) \underset{\cong}{\stackrel{s^{*}}{\leftrightarrows}} H^{q}\left(M_{\mathrm{dR}}^{\bullet}\right),
$$

we obtain a long exact sequence

$\rightarrow H^{q}\left(\Gamma\left(\mathbb{K}, M^{\bullet}(n)\right)\right) \rightarrow H^{q}\left(M_{0}^{\bullet}\right) \oplus H^{q}\left(F^{n} M_{\mathrm{dR}}^{\bullet} \stackrel{\eta^{\prime \prime}}{\rightarrow} H^{q}\left(M_{0}^{\bullet}\right) \oplus H^{q}\left(M_{\mathrm{dR}}^{\bullet}\right) \rightarrow\right.$, where $\eta^{\prime \prime}\left(x_{0}, x_{\mathrm{dR}}\right)=\left(p^{-n} \phi\left(x_{0}^{\sigma}\right)-x_{0}, \cos p\left(x_{0} \otimes 1_{K}\right)-x_{\mathrm{dR}}\right)$.

Proposition 2.2.2 (extension formula). With the notation above, there is a canonical morphism of abelian groups

$$
\operatorname{Hom}_{p H D}\left(M^{\bullet}, M^{\prime \bullet}[n]\right) \cong H^{n}\left(\Gamma\left(M^{\bullet}, M^{\prime \bullet}\right)\right) .
$$

In particular, if $M^{\bullet}=M$ and $M^{\mathbf{\prime}^{\bullet}}=M^{\prime}$ are concentrated in degree 0 , then $H^{n}\left(\Gamma\left(M, M^{\prime}\right)\right)=0$ for $n \geq 2$ and $n<0$.

Proof. By the octahedron axiom, we have the following triangle in $D^{b}(A b)$ :

$$
\operatorname{Ker} \psi_{M^{\bullet}, M^{\prime \bullet}} \rightarrow \Gamma\left(M^{\bullet}, M^{\prime \bullet}\right) \rightarrow \operatorname{Coker} \psi_{M^{\bullet}, M^{\prime}}[-1] \stackrel{+}{\rightarrow} .
$$

Its cohomological long exact sequence is

$$
\stackrel{\partial}{\rightarrow} H^{n}\left(\operatorname{Ker} \psi_{M^{\bullet}, M^{\prime}}\right) \rightarrow H^{n}\left(\Gamma\left(M, M^{\prime \bullet}\right)\right) \rightarrow H^{n}\left(\operatorname{Coker} \psi_{M^{\bullet}, M^{\bullet}}[-1]\right) \stackrel{\partial}{\rightarrow} .
$$


Note that by construction, $H^{n}\left(\operatorname{Ker} \psi_{M^{\bullet}, M^{\prime}}\right)=\operatorname{Hom}_{p H K}\left(M^{\bullet}, M^{\prime^{\bullet}}[n]\right)$. Also, we have

$\operatorname{Hom}_{p H D}\left(M^{\bullet}, M^{\prime \bullet}[n]\right)=\underset{I}{\operatorname{colim}} \operatorname{Hom}_{p H K}\left(M^{\bullet}, M^{\prime \prime \bullet}[n]\right), I=\left\{\right.$ quis $\left.g: M^{\prime \bullet} \rightarrow M^{\prime \prime \bullet}\right\}$.

Thus, the result is proved if we show that

(i) $H^{n}\left(\Gamma\left(M^{\bullet}, M^{\prime \bullet}\right)\right) \cong H^{n}\left(\Gamma\left(M^{\bullet}, M^{\prime \prime \bullet}\right)\right)$ holds given any $g: M^{\prime \bullet} \rightarrow M^{\prime \prime \bullet}$ quasiisomorphism and

(ii) $\operatorname{colim}_{I} H^{n}\left(\operatorname{Coker} \psi_{M^{\bullet}, M^{\prime \prime}}[-1]\right)=0$.

The first claim follows from the exactness of $\Gamma\left(M^{\bullet}, \cdot\right)$, and the second is proved in [Beĭlinson 1986, 1.7, 1.8] (and with more details in [Huber 1995, Lemma 4.2.8; Bannai 2002, Lemma 2.15]) with the assumption that all the gluing maps are quasiisomorphisms, but this hypothesis is not necessary.

Lemma 2.2.3 (tensor product). Let $M^{\bullet}, M^{\bullet}$ and $I^{\bullet}$ be p-adic Hodge complexes. For any $\alpha \in K$, there is a morphism of complexes

$$
\cup_{\alpha}: \Gamma\left(I^{\bullet}, M^{\bullet}\right) \otimes \Gamma\left(I^{\bullet}, M^{\prime \bullet}\right) \rightarrow \Gamma\left(I^{\bullet}, M^{\bullet} \otimes M^{\prime \bullet}\right) .
$$

All such $\cup_{\alpha}$ are equivalent up to homotopy.

Proof. See [Beйlinson 1986, 1.11].

Remark 2.2.4 (enlarging the diagram). We recall some results from [Levine 1998, Chapter V, 2.3.3]. Let $M_{1}^{\bullet} \stackrel{f}{\rightarrow} M_{2}^{\bullet} \stackrel{g}{b} M_{3}^{\bullet}$ (resp. $M_{1}^{\bullet} \stackrel{f}{\leftarrow} M_{2}^{\bullet} \stackrel{g}{\rightarrow} M_{3}^{\bullet}$ ) be a diagram of complexes in $C^{b}(K)$. Let $P^{\bullet}=\operatorname{Cone}\left(f-g: M_{1}^{\bullet} \oplus M_{3}^{\bullet} \rightarrow M_{2}^{\bullet}\right)[-1]$ be the quasipullback complex (resp. $Q^{\bullet}=\operatorname{Cone}\left((f,-g): M_{2}^{\bullet} \rightarrow M_{1}^{\bullet} \oplus M_{3}^{\bullet}\right)$ be the quasipushout). Assume that $f$ is a quasi-isomorphism. Then the diagrams
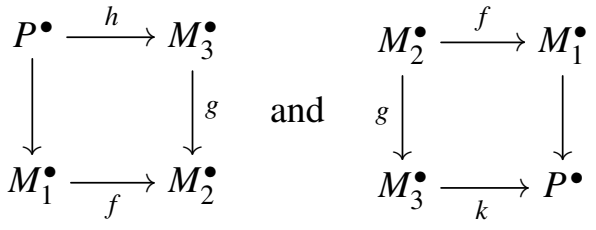

are commutative up to homotopy and are such that $h$ and $k$ are quasi-isomorphisms.

Now let $p H C^{\prime}$ be a category of systems $\left(M_{\mathrm{rig}}^{\bullet}, M_{\mathrm{dR}}^{\bullet}, M_{1}^{\bullet}, M_{2}^{\bullet}, M_{3}^{\bullet}, c, s, f, g\right)$ similar to Definition 2.0.11 and such that there is a diagram

$$
M_{\text {rig }}^{\bullet} \stackrel{c}{\rightarrow} M_{1}^{\bullet} \stackrel{f}{\leftarrow} M_{2}^{\bullet} \stackrel{g}{\rightarrow} M_{3}^{\bullet} \stackrel{s}{\leftarrow} M_{\mathrm{dR}}^{\bullet} .
$$

Then the quasipushout induces a functor from the category $p H C^{\prime}$ to the category $p H C$. This functor is compatible with tensor product after passing to the categories $p H K^{\prime}$ and $p H K$. 


\section{Godement resolution}

Here we recall some facts about the generalized Godement resolution, also called the bar-resolution. We refer to [Ivorra 2005]; see also [Weibel 1994, Section 8.6].

3.1. General construction. Let $u: P \rightarrow X$ be a morphism of Grothendieck topologies so that $P^{\sim}$ (resp. $X^{\sim}$ ) is the category of abelian sheaves on $P$ (resp. $X$ ). Then we have a pair of adjoint functors $\left(u^{*}, u_{*}\right)$, where $u^{*}: X^{\sim} \rightarrow P^{\sim}$ and $u_{*}: P^{\sim} \rightarrow X^{\sim}$. For any object $\mathscr{F}_{F}$ of $X^{\sim}$, we can define a cosimplicial object $B^{*}(\mathscr{F}): \Delta \rightarrow X^{\sim}$ in the following way. First let $\eta: \operatorname{id}_{X^{\sim}} \rightarrow u_{*} u^{*}$ and $\epsilon: u^{*} u_{*} \rightarrow \operatorname{id}_{P^{\sim}}$ be the natural transformations induced by adjunction.

Endow $B^{n}(\mathscr{F}):=\left(u_{*} u^{*}\right)^{n+1}(\mathscr{F})$ with codegeneracy maps

$$
\sigma_{i}^{n}:=\left(u_{*} u^{*}\right)^{i} u_{*} \in u^{*}\left(u_{*} u^{*}\right)^{n-1-i}: B^{n}(\mathscr{F}) \rightarrow B^{n-1}(\mathscr{F}) \text { for } i=0, \ldots, n-1
$$

and cofaces

$$
\delta_{i}^{n-1}:=\left(u_{*} u^{*}\right)^{i} \eta\left(u_{*} u^{*}\right)^{n-i}: B^{n-1}(\mathscr{F}) \rightarrow B^{n}(\mathscr{F}) \quad \text { for } i=0, \ldots, n .
$$

Lemma 3.1.1. With the notation above, let $s B^{*}(\mathscr{F})$ be the associated complex of objects of $X^{\sim}$. Then there is a canonical map $b_{\mathscr{F}}: \mathscr{F} \rightarrow s B^{*}\left(\mathscr{F}_{F}\right)$ such that $u^{*}\left(b_{\mathscr{F}}\right)$ is a quasi-isomorphism. Moreover, if $u^{*}$ is exact and conservative, then $b_{\mathscr{F}}$ is a quasi-isomorphism.

Proof. See [Ivorra 2005, Chapter III, Lemma 3.4.1].

Thus, for any sheaf $\mathscr{F} \in X^{\sim}$ (or complex of sheaves), we can define a functorial map $b_{\mathscr{F}}: \mathscr{F} \rightarrow s B^{*}(\mathscr{F})$ with $s B^{n}(\mathscr{F}):=\left(u_{*} u^{*}\right)^{n+1} \mathscr{F}$. We will denote this complex of sheaves $\operatorname{Gd}_{P}(\mathscr{F})$. In the case $u^{*}$ is exact and conservative, $\operatorname{Gd}_{P}(\mathscr{F})$ is a canonical

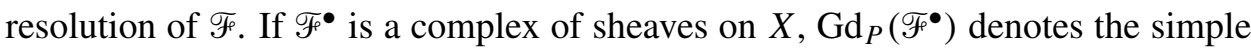
complex $s\left(\operatorname{Gd}_{P}\left(\mathscr{F}^{i}\right)^{j}\right)$. Often, we will need to iterate this process, and we will write $\operatorname{Gd}_{P}^{2}(\mathscr{F}):=\operatorname{Gd}_{P}\left(\operatorname{Gd}_{P}(\mathscr{F})\right)$.

Now suppose there is a commutative diagram of sites

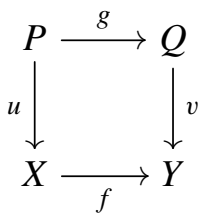

and a morphism of sheaves $a: \mathscr{G} \rightarrow f_{*} \mathscr{F}$, where $\mathscr{F}($ resp. $\mathscr{G})$ is a sheaf on $X($ resp. $Y$ ). Lemma 3.1.2. There is a canonical map $\operatorname{Gd}_{Q}(\mathscr{G}) \rightarrow f_{*} \operatorname{Gd}_{P}(\mathscr{F})$ compatible with $b_{\mathscr{F}}$ and $b$.

Proof. We need only show that there is a canonical map $v_{*} v^{*} G_{G} \rightarrow f_{*} u_{*} u^{*} \mathscr{F}$ lifting $a$. First consider the composition $\mathscr{G} \rightarrow f_{*} \mathscr{F} \rightarrow f_{*} u_{*} u^{*} \mathscr{F}$. Then we get a 
map $\mathscr{G}_{\rightarrow} \rightarrow v_{*} g_{*} u^{*} \mathscr{F}$ because $v_{*} g_{*}=f_{*} u_{*}$. By adjunction, this gives $v^{*} \mathscr{G}_{\rightarrow} \rightarrow g_{*} u^{*} \mathscr{F}$. Then we apply $v_{*}$ and use $v_{*} g_{*}=f_{*} u_{*}$ to obtain the desired map.

Remark 3.1.3 (tensor product). The Godement resolution is compatible with tensor products; that is, for any pair of sheaves $\mathscr{F}$ and $\mathscr{G}$ on $X$, there is a canonical quasiisomorphism $\operatorname{Gd}_{P}(\mathscr{F}) \otimes \operatorname{Gd}_{P}(\mathscr{G}) \rightarrow \operatorname{Gd}_{P}(\mathscr{F} \otimes \mathscr{G})$. The same holds for complexes that are bounded below [Friedlander and Suslin 2002, Appendix A].

\subsection{Points of sites/topoi.}

Definition 3.2.1 (see [Artin et al. 1972a, Example IV, Section 6]). Let $X$ be a site and $\operatorname{Sh}(X)$ be the associated topos of sheaves of sets. A point of $X$ is a morphism of topoi $\pi: \operatorname{Set} \rightarrow \operatorname{Sh}(X)$, that is, a pair of adjoint functors $\left(\pi^{*}, \pi_{*}\right)$ such that $\pi^{*}$ is left exact.

Example 3.2.2. Let $X$ be a scheme. Then any point $x$ of the topological space underlying $X$ gives a point $\pi_{x}$ of the Zariski site of $X$. We call them Zariski points.

Now let $x$ be a geometric point of $X$; then it induces a point $\pi_{x}$ for the étale site of $X$. We call them étale points of $X$.

Let $\mathscr{F}$ be a Zariski (resp. étale) sheaf $X$ and $P$ be the set of Zariski (resp. étale) points of $X$. Then the functor $\mathscr{F}_{F} \mapsto \bigsqcup_{\pi \in P} \mathscr{F}_{\pi}:=\pi^{*} \mathscr{F}$ is exact and conservative. In other words, the Zariski (resp. étale) site of $X$ has enough points.

Example 3.2.3 (points on rigid analytic spaces [van der Put and Schneider 1995]). Let $\mathscr{X}$ be a rigid analytic space over $K$. We recall that a filter $f$ on $\mathscr{L}$ is a collection $\left(U_{\alpha}\right)_{\alpha}$ of admissible open subsets of $X$ satisfying

(i) $\mathscr{X} \in f$ and $\varnothing \notin f$,

(ii) if $u_{\alpha}, u_{\beta} \in f$, then $u_{\alpha} \cap u_{\beta} \in f$ and

(iii) if $\mathcal{U}_{\alpha} \in f$ and the admissible open $\mathscr{V}$ contains $\boldsymbol{U}_{\alpha}$, then $\mathscr{V} \in f$.

A prime filter on $\mathscr{L}$ is a filter $p$ satisfying moreover

(iv) if $u \in p$ and $u=\bigcup_{i \in I} u_{i}^{\prime}$ is an admissible covering of $u$, then $u_{i_{0}}^{\prime} \in p$ for some $i_{0} \in I$.

Let $P(\mathscr{X})$ be the set of all prime filters of $\mathscr{X}$. The filters on $\mathscr{X}$ are ordered with respect to inclusion. We can give to $P(\mathscr{C})$ a topology and define a morphism of sites $\sigma: P(\mathscr{X}) \rightarrow \mathscr{X}$. Also, we let $P t(\mathscr{X})$ denote the set of prime filters with the discrete topology. Let $i: P t(\mathscr{X}) \rightarrow P(X)$ be the canonical inclusion and $\xi=\sigma \circ i$.

Remark 3.2.4. Let $p=\left(U_{\alpha}\right)_{\alpha}$ be a prime filter on $\mathscr{X}$ as above. Then $p$ is a point of the site $\mathscr{X}$ (see Definition 3.2.1). Using the construction of the continuous map $\sigma$ of [van der Put and Schneider 1995], we get that the morphism of topoi $\pi: \operatorname{Set} \rightarrow \operatorname{Sh}(\mathscr{X})$, 
associated to $p$, is defined in the following way. For any sheaf (of sets) $\mathscr{F}$ on $\mathscr{X}$, let $\pi^{*}(\mathscr{F})=\operatorname{colim}_{\alpha} \mathscr{F}\left(\mathcal{U}_{\alpha}\right)$; for any set $\mathscr{Y}$ and $\mathscr{V}$ admissible open in $\mathscr{X}$, let

$$
\pi_{*} \mathscr{Y}(\mathscr{V})= \begin{cases}\mathscr{Y} & \text { if } \mathscr{V}=\mathcal{U}_{\alpha} \text { for some } \alpha \\ 0 & \text { otherwise }\end{cases}
$$

where 0 denotes the final object in the category Set. In fact, with the notation above, we easily get the adjunction

$$
\operatorname{Hom}_{S e t}\left(\pi^{*}(\mathscr{F}), \mathscr{S}\right)=\lim _{\alpha} \operatorname{Hom}_{S e t}\left(\mathscr{F}\left(\mathcal{U}_{\alpha}\right), \mathscr{S}\right)=\operatorname{Hom}_{S h(\mathscr{C})}\left(\mathscr{F}, \pi_{*} \mathscr{Y}\right) .
$$

Lemma 3.2.5. With the notation above, the functor $\xi^{-1}: \operatorname{Sh}(\mathscr{X}) \rightarrow \operatorname{Sh}(\operatorname{Pt}(\mathscr{X}))$ is exact and conservative. In other words, for any $p \in P t(\mathscr{Q}), \mathscr{F}_{F}=0$ if all $\mathscr{F}_{p}=0$ and the functors $\operatorname{Sh}(\mathscr{X}) \ni \mathscr{F}_{F} \mapsto \mathscr{F}_{p}$ are exact.

Proof. See [van der Put and Schneider 1995, Section 4] after the proof of Theorem 1.

\section{Rigid and de Rham complexes}

We begin this section by recalling the construction of the rigid complexes of [Besser 2000, Section 4]. Instead of the techniques of [Artin et al. 1972b, Exposé V.bis], we use the machinery of generalized Godement resolution as developed in Section 3. This alternative approach was also mentioned by Besser in the introduction of his paper. We then recall the construction of the de Rham complexes.

4.1. Rigid complexes. We define a rigid triple to be a system $(X, \bar{X}, \mathrm{P})$, where $X$ is an algebraic $k$-scheme, $j: X \rightarrow \bar{X}$ is an open embedding into a proper $k$-scheme and $\bar{X} \rightarrow \mathrm{P}$ is a closed embedding into a $p$-adic formal $\mathscr{V}$-scheme $\mathrm{P}$ that is smooth in a neighborhood of $X$.

Definition 4.1.1 [Besser 2000, 4.2, 4.4]. Let $(X, \bar{X}, \mathrm{P})$ and $(Y, \bar{Y}, \mathrm{Q})$ be two rigid triples, and let $f: X \rightarrow Y$ be a morphism of $k$-schemes. Let $U \subset] \bar{X}\left[{ }_{P}\right.$ be a strict neighborhood of $] X$ [P and $F: U \rightarrow Q_{K}$ be a morphism of $K$-rigid spaces. We say that $F$ is compatible with $f$ if it induces the following commutative diagram:

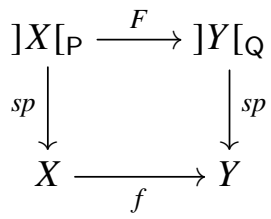

We write $\operatorname{Hom}_{f}\left(U, \mathrm{Q}_{K}\right)$ for the collection of morphisms compatible with $f$.

The collection of rigid triples forms a category RT with the set of pairs $(f, F)$ written as $\operatorname{Hom}((X, \bar{X}, \mathrm{P}),(Y, \bar{Y}, \mathrm{Q}))$, where $f: X \rightarrow Y$ is a $k$-morphism and $F \in \operatorname{colim}_{\imath} \operatorname{Hom}_{f}(u, Q)$. 


\section{Proposition 4.1.2. (i) There is a functor}

$$
(\mathrm{Sch} / k)^{\circ} \rightarrow C\left(K_{0}\right), \quad X \mapsto R \Gamma_{\text {rig }}\left(X / K_{0}\right)
$$

from the category of algebraic $k$-schemes with proper morphisms $\mathrm{Sch} / k$, such that $H^{i}\left(R \Gamma_{\text {rig }}\left(X / K_{0}\right)\right) \cong H_{\text {rig }}^{i}\left(X / K_{0}\right)$. Moreover, a canonical $\sigma$-linear endomorphism of $R \Gamma_{\text {rig }}\left(X / K_{0}\right)$ exists inducing the Frobenius on cohomology.

(ii) There are two functors $\mathrm{RT} \rightarrow C(K)$

$$
\widetilde{R \Gamma}_{\text {rig }}(X)_{\bar{X}, \mathrm{P}} \text { and } R \Gamma_{\text {rig }}(X / K)_{\bar{X}, \mathrm{P}}
$$

and functorial quasi-isomorphisms with respect to maps of rigid triples

$$
R \Gamma_{\text {rig }}(X / K) \leftarrow \widetilde{R \Gamma}_{\text {rig }}(X)_{\bar{X}, \mathrm{P}} \rightarrow R \Gamma_{\text {rig }}(X)_{\bar{X}, \mathrm{P}}
$$

Proof. See [Besser 2000, 4.9, 4.21, 4.22].

Remark 4.1.3. The building block of the construction is the functor $R \Gamma_{\text {rig }}(X / K)_{\bar{X}, \mathrm{P}}$. That complex is constructed with a system of compatible resolution of the overconvergent de Rham complexes $j_{X}^{\dagger} \Omega_{U}^{\bullet}$, where $U$ runs over all strict neighborhoods of the tube of $X$. Using Godement resolution, we can explicitly define

$$
R \Gamma_{\text {rig }}(X / K)_{\bar{X}, \mathrm{P}}:=\operatorname{colim}_{U} \Gamma\left(U, \operatorname{Gd}_{\mathrm{an}} j_{X}^{\dagger} \operatorname{Gd}_{\mathrm{an}} \Omega_{\mathscr{U}}^{\bullet}\right),
$$

where $\mathrm{Gd}_{\mathrm{an}}=\mathrm{Gd}_{P t(U)}$. This will be an essential ingredient for achieving the main results of the paper.

All the proofs of [Besser 2000, Section 4] work using this Godement complex. We recall that

$$
\begin{aligned}
& R \Gamma_{\text {rig }}(X / K):=\operatorname{colim}_{A \in \mathrm{SET}_{X}^{0}} R \Gamma_{\text {rig }}(X / K)_{\bar{X}_{A}, \mathrm{P}_{A},} \\
& \widetilde{R \Gamma}_{\text {rig }}(X / K):=\operatorname{colim}_{A \in \mathrm{SET}_{(X, \bar{X}, \mathrm{P})}^{0}} R \Gamma_{\text {rig }}(X / K)_{\bar{X}_{A}, \mathrm{P}_{A}},
\end{aligned}
$$

where $\mathrm{SET}_{X}^{0}$ and $\mathrm{SET}_{(X, \bar{X}, \mathrm{P})}^{0}$ are filtered categories of indexes.

With some modifications, we can provide a compact support version of the functors above. We just need to be careful in the choice of morphisms of rigid triples.

Definition 4.1.4. Let $(X, \bar{X}, \mathrm{P})$ and $(Y, \bar{Y}, \mathrm{Q})$ be two rigid triples, and let $f: X \rightarrow Y$ be a morphism of $k$-schemes. Let $F: \cup \rightarrow Q_{K}$ be compatible with $f$ (as in 
Definition 4.1.1). We say that $F$ is strict if there is a commutative diagram

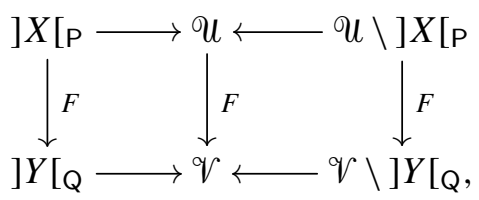

where $\mathscr{V}$ is a strict neighborhood of $] Y{ }_{\mathrm{Q}}$ in $] \bar{Y}[\mathrm{Q}$.

It is easy to show that strict morphisms are composable. We let $\mathrm{RT}_{c}$ denote the category of rigid triples with proper morphisms, which is the (not full) subcategory of RT with the same objects and morphisms pairs $(f, F)$, where $f$ is proper and $F$ is a germ of a strict compatible morphism.

Lemma 4.1.5. (i) Let $(X, \bar{X}, \mathrm{P})$ be a rigid triple, and let $u$ be a strict neighborhood of $] X[p$. Then

$$
H^{i}\left(\Gamma\left(\mathcal{U}, \operatorname{Gd}_{\mathrm{an}} \underline{\Gamma}_{] X[\mathrm{P}} \operatorname{Gd}_{\mathrm{an}} \Omega_{\ddot{\vartheta}}^{\bullet}\right)\right)=H_{\mathrm{rig}, c}^{i}(X) .
$$

(ii) Let $(Y, \bar{Y}, \mathrm{Q})$ be another rigid triple, $f: X \rightarrow Y$ be a proper $k$-morphism and $F: \mathcal{U} \rightarrow Q_{K}$ be a morphism of $K$-analytic space compatible with $f$ and strict. Then there is a canonical map

$$
F^{*}: \operatorname{Gd}_{\mathrm{an}} \underline{\Gamma}_{] Y[\mathrm{Q}} \mathrm{Gd}_{\mathrm{an}} \Omega_{\mathrm{Q}_{K}}^{\bullet} \rightarrow F_{*} \mathrm{Gd}_{\mathrm{an}} \underline{\Gamma}_{] X[\mathrm{P}} \operatorname{Gd}_{\mathrm{an}} \Omega_{u}^{\bullet} .
$$

Proof. (i) It is sufficient to note that $\operatorname{Gd}_{\mathrm{an}}\left(\Omega_{u}^{\bullet}\right)$ is a complex of flasque sheaves and that a flasque sheaf is acyclic for $\underline{\Gamma}_{] X[\mathrm{p}}$. Let $F$ be a flasque sheaf on the rigid analytic space $U$. By definition, $\underline{\Gamma}_{] X[\mathrm{p}}=\operatorname{Ker}\left(a: F \rightarrow i_{*} i^{*} F\right)$. It is easy to check that $\mathbb{R}^{q} i_{*} i^{*} F=0$ for $q \geq 1$. Hence, $\mathbb{R} \Gamma_{] X[\mathrm{P}} F \cong \operatorname{Cone}\left(a: F \rightarrow i_{*} i^{*} F\right)[-1]$. But by hypothesis, the map $a$ is surjective, so $\operatorname{Cone}\left(a: F \rightarrow i_{*} i^{*} F\right)[-1] \cong \operatorname{Ker}(a)=\mathbb{R}^{0} \underline{\Gamma}_{] X[\mathrm{p}}$. (ii) First consider the canonical pullback of differential forms $F^{*}: \Omega_{\mathrm{Q}_{K}} \rightarrow F_{*} \Omega_{\vartheta}$. Then by Lemma 3.1.2, we get a map $\operatorname{Gd}_{P t\left(\mathrm{Q}_{K}\right)} \Omega_{\mathrm{Q}_{K}}^{\bullet} \rightarrow F_{*} \operatorname{Gd}_{P t(\vartheta)} \Omega_{U}$. Applying the functor $\underline{\Gamma}_{] X[\mathrm{P}}$ to the adjoint map, we get

$$
\underline{\Gamma}_{] X[\mathrm{P}} F^{-1} \mathrm{Gd}_{P t\left(\mathrm{Q}_{K}\right)} \Omega_{\mathrm{Q}_{K}} \rightarrow \underline{\Gamma}_{] X[\mathrm{P}} \mathrm{Gd}_{P t(\vartheta)} \Omega_{u} .
$$

But the strictness of $F$ implies that there is a canonical map $F^{-1} \underline{\Gamma}_{] Y[\mathrm{Q}} \rightarrow \underline{\Gamma}_{] X[\mathrm{p}} F^{-1}$ [Le Stum 2007, proof of Proposition 5.2.17]. Hence, we have a map

$$
F^{-1} \underline{\Gamma}_{] Y[\mathrm{Q}} \operatorname{Gd}_{P t\left(\mathrm{Q}_{K}\right)} \Omega_{\mathrm{Q}_{K}} \rightarrow \underline{\Gamma}_{] X[\mathrm{P}} \operatorname{Gd}_{P t(U)} \Omega_{\text {थ }}
$$

We can conclude the proof by taking the adjoint of this map and again applying Lemma 3.1.2.

Proposition 4.1.6. (i) There is a functor

$$
\left(\operatorname{Sch}_{c} / k\right)^{\circ} \rightarrow C\left(K_{0}\right), \quad X \mapsto R \Gamma_{\text {rig }, c}\left(X / K_{0}\right)
$$


from the category of algebraic $k$-schemes with proper morphisms $\mathrm{Sch}_{c} / k$, such that $H^{i}\left(R \Gamma_{\mathrm{rig}, c}\left(X / K_{0}\right)\right) \cong H_{\mathrm{rig}, c}^{i}\left(X / K_{0}\right)$. Moreover, there exists a canonical $\sigma$-linear endomorphism of $R \Gamma_{\text {rig, }, c}\left(X / K_{0}\right)$ inducing the Frobenius on cohomology.

(ii) There are two functors $\mathrm{RT}_{c} \rightarrow C(K)$

$$
\widetilde{R \Gamma}_{\text {rig }, c}(X)_{\bar{X}, \mathrm{P}} \quad \text { and } \quad R \Gamma_{\text {rig }, c}(X / K)_{\bar{X}, \mathrm{P}}
$$

and functorial quasi-isomorphisms with respect to maps of rigid triples

$$
R \Gamma_{\text {rig }, c}(X / K) \leftarrow \widetilde{R \Gamma}_{\text {rig }, c}(X)_{\bar{X}, \mathrm{P}} \rightarrow R \Gamma_{\text {rig }, c}(X)_{\bar{X}, \mathrm{P}} .
$$

Proof. In view of Lemma 4.1.5, it suffices to mimic the construction given in [Besser 2000, 4.9, 4.21, 4.22] but using only proper morphisms of $k$-schemes and strict compatible maps. In this case, the functors used in the construction are

$$
\begin{aligned}
R \Gamma_{\text {rig }, c}(X / K)_{\bar{X}, \mathrm{P}} & :=\operatorname{colim}_{\mathscr{U}} \Gamma\left(U, \operatorname{Gd}_{\mathrm{an}} \underline{\Gamma}_{] X{ }_{\mathrm{P}}} \operatorname{Gd}_{\mathrm{an}} \Omega_{\vartheta}^{\bullet}\right), \\
R \Gamma_{\text {rig }, c}(X / K) & :=\operatorname{colim}_{A \in \mathrm{SET}_{X}^{0}} R \Gamma_{\text {rig }, c}(X / K)_{\bar{X}_{A}, \mathrm{P}_{A}}, \\
\widetilde{R \Gamma}_{\text {rig }, c}(X / K) & :=\operatorname{colim}_{A \in \mathrm{SET}_{(X, \bar{X}, \mathrm{P})}^{0}} R \Gamma_{\text {rig }, c}(X / K)_{\bar{X}_{A}, \mathrm{P}_{A}} .
\end{aligned}
$$

4.2. de Rham complexes. Now we focus on de Rham complexes, and we deal with smooth $K$-algebraic schemes. Let $X$ be a smooth algebraic $K$-scheme. The (algebraic) de Rham cohomology of $X$ is the hypercohomology of its complex of Kähler differentials $H_{\mathrm{dR}}^{i}(X / K):=H^{i}\left(X, \Omega_{X / K}^{\bullet}\right)$ [Grothendieck 1966b]. We can also define the de Rham cohomology with compact support [Baldassarri et al. 2004, Section 1] as the hypercohomology groups $H_{d R, c}^{i}(X / K):=H^{i}\left(\bar{X}, \lim _{n} J^{n} \Omega_{\bar{X} / K}^{\bullet}\right)$, where $X \rightarrow \bar{X}$ is a smooth compactification and $J$ is the sheaf of ideals associated to the complement $\bar{X} \backslash X$ (this definition does not depend on the choice of $\bar{X}$ [Baldassarri et al. 2004, Theorem 1.8]). In order to consider the Hodge filtration on the de Rham cohomology groups, we fix a normal crossings compactification $g: X \rightarrow Y$ and let $D:=Y \backslash X$ be the complement divisor (this is possible by the Nagata compactification theorem and the Hironaka resolution theorem [Deligne 1971, Section 3.2.1]). We let $\Omega_{Y}^{\bullet}\langle D\rangle$ denote the de Rham complex of $Y$ with logarithmic poles along $D$ (in the Zariski topology) [Jannsen 1990, 3.3]. Let $I \subset O_{Y}$ be the defining sheaf of ideals of $D$.

Proposition 4.2.1. With the notation above,

(i) there is a canonical isomorphism

$$
H_{\mathrm{dR}}^{i}(X) \cong H^{i}\left(Y, \Omega_{Y}^{\bullet}\langle D\rangle\right) \quad\left(\text { resp. } H_{\mathrm{dR}, c}^{i}(X) \cong H^{i}\left(Y, I \Omega_{Y}^{\bullet}\langle D\rangle\right)\right),
$$


(ii) the spectral sequence

$$
E_{1}^{p, q}=H^{q}\left(Y, \Omega^{p}\right) \Rightarrow H^{p+q}\left(Y, \Omega^{\bullet}\right)
$$

degenerates at 1 for $\Omega^{\bullet}=\Omega_{Y}^{\bullet}\langle D\rangle$ and $I \Omega_{Y}^{\bullet}\langle D\rangle$ and

(iii) the filtration induced by this spectral sequence on $H_{\mathrm{dR}}^{i}(X)\left(\right.$ resp. $\left.H_{\mathrm{dR}, c}^{i}(X)\right)$ is independent of the choice of Y. Namely,

$$
F^{j} H_{\mathrm{dR}}^{i}(X):=H^{i}\left(Y, \sigma^{\geq j} \Omega_{Y}^{\bullet}\langle D\rangle\right) \quad\left(\operatorname{resp} . H_{\mathrm{dR}, c}^{i}(X):=H^{i}\left(Y, \sigma^{\geq j} I \Omega_{Y}^{\bullet}\langle D\rangle\right)\right),
$$

where $\sigma^{\geq j}$ is the stupid filtration.

Proof. Using the argument of [Deligne 1971, 3.2.11], we get the independence of the choice of $Y$. The same holds for $H_{\mathrm{dR}, c}^{i}(X)$.

Since our base field $K$ is of characteristic 0 , we can find an embedding $\tau: K \rightarrow \mathbb{C}$. Then by [GAGA 1955-1956], we get these isomorphisms of filtered vector spaces:

$$
\begin{gathered}
H^{i}\left(Y, \Omega_{Y}^{\bullet}\langle D\rangle\right) \otimes_{K} \mathbb{C} \cong H^{i}\left(Y_{h}, \Omega_{Y_{h}}^{\bullet}\left\langle D_{h}\right\rangle\right) \\
\text { (resp. } \left.H^{i}\left(Y, I \Omega_{Y}^{\bullet}\langle D\rangle\right) \otimes_{K} \mathbb{C} \cong H^{i}\left(Y_{h}, I_{h} \Omega_{Y_{h}}^{\bullet}\left\langle D_{h}\right\rangle\right)\right),
\end{gathered}
$$

where $(\cdot)_{h}$ is the complex analytification functor and $I_{h}$ is the defining sheaf of $D_{h}$. Thus, we conclude by [Deligne 1971, Section 3] (resp. [Peters and Steenbrink 2008, Part II, Example 7.25] for the compact support case).

Remark 4.2.2. The degeneracy of the spectral sequence in (ii) of the proposition above can be proved algebraically [Deligne and Illusie 1987]. We don't know an algebraic proof of the isomorphism in (i).

In the sequel, a morphism of pairs $(X, Y)$ as above is a commutative square

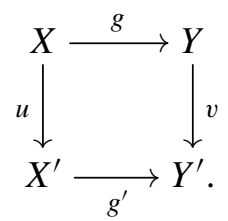

We say that the morphism is strict if the square is cartesian.

The complex $\Omega_{Y}^{\bullet}\langle D\rangle$ (resp. $I \Omega_{Y}^{\bullet}\langle D\rangle$ ) is a complex of Zariski sheaves over $Y$ functorial with respect to the pair $(X, Y)$ (resp. strict morphisms of pairs). We can construct two different (generalized) Godement resolutions (see Section 3): one using Zariski points and the other via the $K$-analytic space associated to $Y$.

We will write $\operatorname{Pt}(Y)=\operatorname{Pt}\left(Y_{\text {zar }}\right)$ for the set of Zariski points of $Y$ with the discrete topology and $P t\left(Y_{\text {an }}\right)$ for the discrete site of rigid points (Example 3.2.3) of $Y_{\text {an }}$; $\operatorname{Pt}\left(Y_{\mathrm{an}}\right) \sqcup \operatorname{Pt}(Y)$ is the direct sum in the category of sites. 
Proposition 4.2.3. With the notation of Proposition 4.2.1, let $w: Y_{\mathrm{an}} \rightarrow Y_{\mathrm{zar}}$ be the canonical map from the rigid analytic site to the Zariski site of $Y$. Then for any Zariski sheaf $\Omega$ on $Y$, there is a diagram

$$
\operatorname{Gd}_{P t(Y)}(\Omega) \leftarrow \operatorname{Gd}_{P t\left(Y_{\text {an }}\right) \sqcup P t(Y)}(\Omega) \rightarrow w_{*} \operatorname{Gd}_{P t\left(Y_{\text {an }}\right)}\left(w^{*} \Omega\right) .
$$

If we further consider $\Omega=\Omega_{Y}^{\bullet}\langle D\rangle$ (resp. $\Omega=I \Omega_{Y}^{\bullet}\langle D\rangle$ ), then the diagram is functorial with respect to the pair $(X, Y)$ (resp. $(X, Y)$ and strict morphisms). The same holds true with $\mathrm{Gd}_{\text {? }}^{2}$ instead of $\mathrm{Gd}_{\text {? }}$.

Proof. The first claim follows from Lemma 3.1.2 applied to the commutative diagram of sites

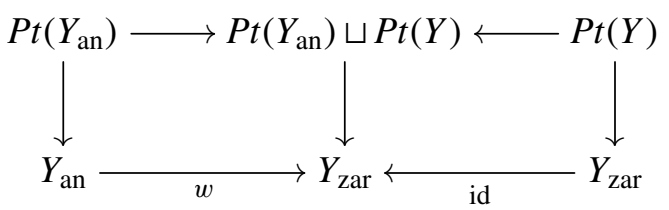

with respect to the canonical map $\Omega \rightarrow w_{*} w^{*} \Omega$. The second claim follows from the functoriality of the complex $\Omega_{Y}^{\bullet}\langle D\rangle$ (resp. $I \Omega_{Y}^{\bullet}\langle D\rangle$ ).

Proposition 4.2.4. (i) Let $g: X \rightarrow Y$ be a normal crossings compactification as in Section 4.2. Then there is a quasi-isomorphism of complexes of sheaves

$$
\Omega_{Y}^{\bullet}\langle D\rangle \rightarrow \operatorname{Gd}_{P t(Y)}^{2}\left(\Omega_{Y}^{\bullet}\langle D\rangle\right) \quad\left(\text { resp. } I \Omega_{Y}^{\bullet}\langle D\rangle \rightarrow \operatorname{Gd}_{P t(Y)}^{2} I \Omega_{Y}^{\bullet}\langle D\rangle\right),
$$

and the stupid filtration on $\Omega_{Y}^{\bullet}\langle D\rangle\left(\right.$ resp. $\left.I \Omega_{Y}^{\bullet}\langle D\rangle\right)$ induces a filtration on the right term of the morphism.

(ii) Let $\mathrm{Sm} / K$ (resp. $\mathrm{Sm}_{c} / K$ ) be the category of algebraic and smooth $K$-schemes (resp. with proper morphisms). Let $D_{\mathrm{dR}}^{b}(K)$ be the derived category of the exact category of filtered vector spaces. Then there exist two functors

$$
R \Gamma_{\mathrm{dR}}(\cdot):(\mathrm{Sm} / K)^{\circ} \rightarrow D_{\mathrm{dR}}^{b}(K) \quad \text { and } \quad R \Gamma_{\mathrm{dR}, c}(\cdot):\left(\operatorname{Sm}_{c} / K\right)^{\circ} \rightarrow D_{\mathrm{dR}}^{b}(K)
$$

such that with the same notation as $(\mathrm{i}), R \Gamma_{\mathrm{dR}}(X)=\Gamma\left(Y, \operatorname{Gd}_{P t(Y)}^{2}\left(\Omega_{Y}^{\bullet}\langle D\rangle\right)\right)$ and $R \Gamma_{\mathrm{dR}, c}(X)=\Gamma\left(Y, \mathrm{Gd}_{P t(Y)}^{2} I \Omega_{Y}^{\bullet}\langle D\rangle\right)$.

(iii) The filtered complexes $R \Gamma_{\mathrm{dR}}(X)$ and $R \Gamma_{\mathrm{dR}, c}(X)$ are strict (see Remark 2.0.10).

Proof. (i) This follows directly from the definition of Godement resolution.

(ii) This follows from the functoriality of the Godement resolution with respect to morphism of pairs and [Deligne 1971, 3.2.11] or [Huber 1995, Lemma 15.2.3] for the compact support case.

(iii) This follows by [Peters and Steenbrink 2008, Part II, Section 4.3, Section 7.3.1]. 


\section{Syntomic cohomology}

In this section, we construct the $p$-adic Hodge complexes needed to define the rigid syntomic cohomology groups (also with compact support) for a smooth algebraic $\mathscr{V}$-scheme. The functoriality will be a direct consequence of the construction.

\subsection{Compactifications.}

Lemma 5.1.1 [Grothendieck 1966a, 2.8.5]. Let $f: \mathscr{L} \rightarrow \mathscr{V}$ be a morphism of schemes, and let $Z \subset \mathscr{X}_{K}$ be a closed subscheme of the generic fiber of $\mathscr{X}$. Then there exists a unique closed subscheme $\mathscr{L} \subset \mathscr{X}$ that is flat over $\mathscr{V}$ and satisfies $\mathscr{E}_{K}=Z$. Thus, $\mathscr{L}$ is the schematic closure of $Z$ in $\mathscr{X}$.

Proposition 5.1.2. Let $\mathscr{L}$ be a smooth scheme over $\mathscr{V}$. Then there exists a generic normal crossings compactification, that is, an open embedding $g: \mathscr{X} \rightarrow \mathscr{Y}$ such that

(i) Y is proper over $\mathscr{V}$,

(ii) $\mathscr{Y}_{K}$ is smooth over $K$ and

(iii) $\mathscr{D}_{K} \subset \mathscr{Y}_{K}$ is a normal crossings divisor, where $\mathscr{D}=\mathscr{Y} \backslash \mathscr{X}$.

Proof. First by Nagata [Conrad 2007], there exists an open embedding $\mathscr{X}_{K} \rightarrow Y$, where $Y$ is a proper $K$-scheme. By the Hironaka resolution theorem, we can assume that $Y$ is a smooth compactification of $\mathscr{X}_{K}$ with complement a normal crossings divisor. Now we can define $\mathscr{Y}^{\prime}$ to be the gluing of $\mathscr{X}$ and $Y$ along the common open subscheme $\mathscr{X}_{K}$. These schemes are all of finite type over $\mathscr{V}$. It follows from the construction that $\mathcal{Y}^{\prime}$ is a scheme, separated and of finite type over $\mathscr{V}$, whose generic fiber is $Y$.

The Nagata compactification theorem works also in a relative setting, namely for a separated and finite type morphism; hence, we can find a $\mathscr{V}$-scheme $\mathscr{Y}$ that is a compactification of $\mathcal{Y}^{\prime}$ over $\mathscr{V}$. Thus, we get an open and dense embedding $h: \mathscr{y}_{K}^{\prime}=Y \rightarrow \mathscr{y}_{K}$ of proper $K$-schemes, so $h$ is the identity, and the statement is proven.

Remark 5.1.3. We can also give another proof of the previous proposition assuming an embedding in a smooth $\mathscr{V}$-scheme. First by Nagata [Conrad 2007], there exists an open embedding $\mathscr{X} \rightarrow \overline{\mathscr{X}}$. Now assume that $\overline{\mathscr{X}}$ is embeddable in a smooth $\mathscr{V}$ scheme $W$; then by [Włodarczyk 2005, Theorem 1.0.2], we can get a resolution of the $K$-scheme $\overline{\mathscr{X}}_{K}$ by making a sequence of blowups with respect to a family of closed subsets in good position with respect to the regular locus of $\overline{\mathscr{X}}_{K}$, in particular $Z_{i} \cap \mathscr{X}_{K}=\varnothing$. One can perform the same construction directly over $\mathscr{V}$, replacing the closed $Z_{i} \subset \mathscr{W}_{K}$ with their Zariski closure $\mathscr{L}_{i}$ in $\mathscr{W}$. By hypothesis, $Z_{i} \subset \mathscr{W}_{K} \backslash \mathscr{X}_{K}$; hence, its closure $\mathscr{E}_{i}$ is contained in $\mathscr{W} \backslash \mathscr{X}$, and $\left(\mathscr{E}_{i}\right)_{K}=Z_{i}$ by Lemma 5.1.1. The construction doesn't affect what happens on the generic fiber because the blowup 
construction is local and behaves well with respect to open immersions [Hartshorne 1977, Chapter II, 7.15]. This will give 9 as in the proposition.

5.2. Connecting maps. From now on, we keep the notation of Proposition 5.1.2 with $g: \mathscr{L} \rightarrow \mathscr{Y}$ being fixed. To simplify the notation, $\mathscr{X}$ (resp. $\mathscr{Y}$ ) denotes the rigid analytic space associated to the generic fiber of $\mathscr{X}$ (resp. $\mathscr{Y}$ ), usually denoted $\mathscr{X}_{K}^{\text {an }}$. Let $w: \mathcal{Y} \rightarrow\left(\mathscr{Y}_{K}\right)_{\mathrm{zar}}$ be the canonical map of sites.

Sometimes we will simply write $\mathrm{Gd}_{\mathrm{an}}=\mathrm{Gd}_{P t(U)}$ if the rigid space $U$ is clear from the context. Similarly, we write $\mathrm{Gd}_{\mathrm{zar}}=\mathrm{Gd}_{P t(X)}$ to denote the Godement resolution with respect to Zariski points of a $K$-scheme $X$.

Lemma 5.2.1. With the notation above, we have the following morphisms of complexes of $K$-vector spaces:

$\Gamma\left(\mathscr{Y}, \mathrm{Gd}_{\mathrm{an}} j^{\dagger} \mathrm{Gd}_{\mathrm{an}} w^{*} \Omega_{\mathscr{y}_{K}}^{\bullet}\left\langle\mathscr{D}_{K}\right\rangle\right) \rightarrow \Gamma\left(\mathscr{X}, \mathrm{Gd}_{\mathrm{an}} j^{\dagger} \mathrm{Gd}_{\mathrm{an}} \Omega_{\mathscr{X}}^{\bullet}\right) \rightarrow R \Gamma_{\mathrm{rig}}\left(\mathscr{L}_{k} / K\right)_{\mathscr{y}_{k}, \widehat{y}}$, $\Gamma\left(\mathscr{Y}, \operatorname{Gd}_{\mathrm{an}} \Gamma_{] \mathscr{L}_{k}[} \operatorname{Gd}_{\mathrm{an}} w^{*} I \Omega_{\mathscr{y}_{K}}^{\bullet}\left\langle\mathscr{D}_{K}\right\rangle\right)$

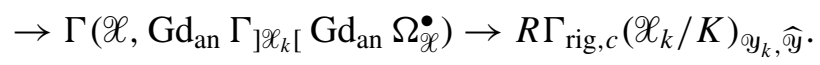

All the maps are quasi-isomorphisms. We let $a$ and $b$ denote the composition of the maps in the first and second diagrams, respectively.

Proof. By construction, $R \Gamma_{\text {rig }}\left(\mathscr{X}_{k} / K\right)_{\mathscr{y}_{k}, \widehat{y}}$ (respectively $\left.R \Gamma_{\text {rig, }, c}\left(\mathscr{X}_{k} / K\right)_{\mathscr{y}_{k}, \widehat{Y}}\right)$ is a direct limit of complexes indexed over the strict neighborhoods of $] \mathscr{L}_{k}[\hat{y}$, and $\mathscr{X}$ is one of them (see Remark 4.1.3 and Proposition 4.1.6). Hence, the map on the right (of both diagrams) comes from the universal property of the direct limit.

For the map on the left, consider first the canonical inclusion of algebraic differential forms with $\log$ poles into the analytic ones $w^{*} \Omega_{\mathscr{y}_{K}}^{\bullet}\left\langle\mathscr{D}_{K}\right\rangle \rightarrow g_{*}^{\text {an }} \Omega_{\mathscr{\mathscr { L }}}^{\bullet}$. By Lemma 3.1.2, we get a map

$$
\operatorname{Gd}_{P t(\mathscr{y})} w^{*} \Omega_{\mathscr{y}_{K}}^{\bullet}\left\langle\mathscr{D}_{K}\right\rangle \rightarrow g_{*}^{\text {an }} \operatorname{Gd}_{P t(\mathscr{C})} \Omega_{\mathscr{\not}}^{\bullet} .
$$

Then applying the $j^{\dagger}$ functor and noting that $j^{\dagger} g_{*}^{\text {an }}=g_{*}^{\text {an }} j^{\dagger}$ [Le Stum 2007, 5.1.14], one obtains a morphism $j^{\dagger} \operatorname{Gd}_{P t(\mathscr{y})} w^{*} \Omega_{\mathscr{Y}_{K}}^{\bullet}\left\langle\mathscr{D}_{K}\right\rangle \rightarrow g_{*}^{\text {an }} j^{\dagger} \operatorname{Gd}_{P t(\mathscr{C})} \Omega_{\mathscr{\mathscr { L }}}^{\bullet}$. This is what we need to apply Lemma 3.1.2 again and conclude the proof for the first diagram.

For the second diagram, repeat the argument using [Le Stum 2007, 5.2.15].

Lemma 5.2.2. With the notation above, we have the following morphisms of complexes of $K$-vector spaces, which we denote by $a^{\prime}$ and $b^{\prime}$, respectively:

$$
\begin{gathered}
\Gamma\left(\mathscr{Y}, \operatorname{Gd}_{\mathrm{an}}^{2} w^{*} \Omega_{\mathscr{y}_{K}}^{\bullet}\left\langle\mathscr{D}_{K}\right\rangle\right) \rightarrow \Gamma\left(\mathscr{Y}, \mathrm{Gd}_{\mathrm{an}} j^{\dagger} \mathrm{Gd}_{\mathrm{an}} w^{*} \Omega_{\mathscr{y}_{K}}^{\bullet}\left\langle\mathscr{D}_{K}\right\rangle\right), \\
\Gamma\left(\mathscr{Y}, \mathrm{Gd}_{\mathrm{an}} \Gamma_{] \mathscr{Q}_{k}[} \operatorname{Gd}_{\mathrm{an}} w^{*} I \Omega_{\mathscr{y}_{K}}^{\bullet}\left\langle\mathscr{D}_{K}\right\rangle\right) \rightarrow \Gamma\left(\mathscr{Y}, \mathrm{Gd}_{\mathrm{an}}^{2} w^{*} I \Omega_{\mathscr{y}_{K}}^{\bullet}\left\langle\mathscr{D}_{K}\right\rangle\right) .
\end{gathered}
$$


Proof. The maps $a^{\prime}$ and $b^{\prime}$ are induced by the canonical maps $\Omega \rightarrow j^{\dagger} \Omega$ and $\Gamma_{] \mathscr{k}_{k}[} \Omega \rightarrow \Omega$, respectively, where $\Omega$ is an abelian sheaf on $y$. In particular, we consider $\Omega=\operatorname{Gd}_{\mathrm{an}} w^{*} \Omega_{\mathscr{O}_{K}}^{\bullet}\left\langle\mathscr{D}_{K}\right\rangle$ (respectively $\Omega=\mathrm{Gd}_{\mathrm{an}} w^{*} I \Omega_{\mathscr{y}_{K}}^{\bullet}\left\langle\mathscr{D}_{K}\right\rangle$ ). To conclude the proof, apply the functor $\mathrm{Gd}_{\mathrm{an}}$ again and take global sections.

5.3. Syntomic complexes. Now we put together all we have done, getting a diagram, say $R \Gamma^{\prime}(\mathscr{C})$, of complexes of $K$-vector spaces:

$$
\begin{aligned}
& R \Gamma_{\text {rig }}\left(\mathscr{X} / K_{0}\right) \longrightarrow \alpha_{\text {rig }}(\mathscr{Q} / K) \\
& \widetilde{R \Gamma}_{\text {rig }}(\mathscr{X} / K)_{\mathscr{y}_{k}, \hat{y}} \stackrel{\alpha_{3}}{\alpha_{4}} R \Gamma_{\text {rig }}(\mathscr{X} / K)_{\mathscr{y}_{k}, \hat{y}} \\
& \Gamma\left(\mathscr{Y}, \mathrm{Gd}_{\mathrm{an}}^{2} w^{*} \Omega \mathscr{g}_{K}\left\langle\mathscr{D}_{K}\right\rangle\right) \underset{\alpha_{5}}{\stackrel{\alpha_{6}}{\longrightarrow}}\left(\mathscr{Y}, \mathrm{Gd}_{\mathrm{an}}^{2} w^{*} \Omega \mathscr{g}_{K}\left\langle\mathscr{D}_{K}\right\rangle\right) \\
& \Gamma\left(\mathscr{Y}_{K}, \mathrm{Gd}_{\mathrm{an}+\mathrm{zar}}^{2} \Omega_{\mathrm{g}_{K}}\left\langle\mathscr{D}_{K}\right\rangle\right) \underset{\alpha_{8}}{\stackrel{\alpha_{7}}{\longrightarrow}} \Gamma\left(\mathscr{y}_{K}, \mathrm{Gd}_{\mathrm{zar}}^{2} \Omega_{\mathrm{g}_{K}}\left\langle\mathscr{D}_{K}\right\rangle\right) \\
& \Gamma\left(\mathscr{Y}_{K}, \mathrm{Gd}_{\mathrm{zar}}^{2} \Omega_{\mathscr{g}_{K}}\left\langle\mathscr{D}_{K}\right\rangle\right)
\end{aligned}
$$

where $\alpha_{1}, \alpha_{5}, \alpha_{8}$ are the identity maps; $\alpha_{2}$ and $\alpha_{3}$ are the maps of Proposition 4.1.2; $\alpha_{4}$ is the composition of $a \circ a^{\prime}$ (see Lemma 5.2.1 and Lemma 5.2.2) and $\alpha_{6}$ and $\alpha_{7}$ are defined in Proposition 4.2.3. By repeatedly applying the quasipushout construction, we obtain a diagram of the shape

$$
R \Gamma_{\text {rig }}\left(\mathscr{X} / K_{0}\right) \rightarrow R \Gamma_{K}(\mathscr{X}) \leftarrow R \Gamma_{\mathrm{dR}}(\mathscr{X}) .
$$

It represents an object of $p H C$ that we denote $R \Gamma(\mathscr{X})$.

Similarly, we can construct a $p$-adic Hodge complex $R \Gamma_{c}(\mathscr{X})$ associated to the diagram $R \Gamma_{c}^{\prime}(\mathscr{L})$ defined as

$$
\begin{aligned}
& R \Gamma_{\text {rig }, c}\left(\mathscr{X} / K_{0}\right) \longrightarrow \frac{\beta_{1}}{\beta_{2} \longrightarrow} R \Gamma_{\text {rig }, c}(\mathscr{X} / K) \\
& \widetilde{R \Gamma}_{\text {rig }, c}(\mathscr{X} / K)_{\mathscr{O}_{k}, \hat{y}} \stackrel{\beta_{3}}{\beta_{4}} R \Gamma_{\text {rig, }, c}(\mathscr{X} / K)_{\mathscr{O}_{k}, \hat{y}}
\end{aligned}
$$

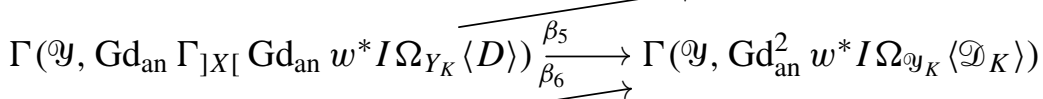

$$
\begin{aligned}
& \Gamma\left(\mathscr{y}_{K}, \mathrm{Gd}_{\mathrm{an}+\mathrm{zar}}^{2} I \Omega_{\mathrm{g}_{K}}\left\langle\mathscr{D}_{K}\right\rangle\right) \stackrel{\overrightarrow{\beta_{7}}}{\longrightarrow} \Gamma\left(\mathscr{Y}_{K}, \mathrm{Gd}_{\mathrm{zar}}^{2} I \Omega_{\mathscr{y}_{K}}\left\langle\mathscr{D}_{K}\right\rangle\right) \\
& \Gamma\left(\mathscr{Y}_{K}, \operatorname{Gd}_{\mathrm{zar}}^{2} I \Omega \mathscr{y}_{K}\left\langle\mathscr{D}_{K}\right\rangle\right)
\end{aligned}
$$

where $\beta_{1}$ and $\beta_{8}$ are the identity maps; $\beta_{2}$ and $\beta_{3}$ are the maps of Proposition 4.1.2; $\beta_{4}$ is the map $b$ of Lemma 5.2.1; $\beta_{5}=b^{\prime}$ of Lemma 5.2.2 and $\beta_{6}$ and $\beta_{7}$ are defined in Proposition 4.2.3. Note that $\beta_{6}$ is a quasi-isomorphism by GAGA.

Proposition 5.3.1. Let $S m / R$ (resp. $S m_{c} / R$ ) be the category of algebraic and smooth $R$-schemes (resp. with proper morphisms). The previous construction 
induces the functors

$$
R \Gamma(\cdot):(\mathrm{Sm} / \mathscr{V})^{\circ} \rightarrow p H D \quad \text { and } \quad R \Gamma_{c}(\cdot):\left(\operatorname{Sm}_{c} / \mathscr{V}\right)^{\circ} \rightarrow p H D .
$$

Proof. Let $f: \mathscr{X} \rightarrow \mathscr{X}^{\prime}$ be a morphism of smooth $\mathscr{V}$-schemes. To get the functoriality, we just have to show that can find two gncd compactifications $g: \mathscr{X} \rightarrow \mathscr{Y}$ and $g^{\prime}: \mathscr{X}^{\prime} \rightarrow \mathcal{Y}^{\prime}$ and a map $h: \mathscr{Y} \rightarrow \mathcal{Y}^{\prime}$ extending $f$, that is, $h g=g^{\prime} f$. We argue as in [Deligne 1971, Section 3.2.11]. Fix two gncd compactifications $g^{\prime}: \mathscr{X}^{\prime} \rightarrow \mathscr{Y}^{\prime}$ and $l: \mathscr{X} \rightarrow \mathscr{L}$. Then consider the canonical map $\mathscr{X} \rightarrow \mathscr{L} \times \mathscr{Y}^{\prime}$ induced by $l$ and $g^{\prime} f$. Let $\overline{\mathscr{X}}$ be the closure of $\mathscr{X}$ in $\mathscr{E} \times \mathscr{Y}^{\prime}$ and use the same argument as in the proof of Proposition 5.1.2 to get 9 , which is generically a resolution of the singularities of $\overline{\mathscr{X}}$. Then by Proposition 4.2.3 and Proposition 4.1.2, we get the functoriality of $R \Gamma(\cdot)$.

If we further assume $f$ to be proper, then we can apply the same argument in order to get the commutative square $h g=g^{\prime} f$ as above. Then by properness, this square is also cartesian by [Huber 1995, Lemma 15.2.3]. From this fact and Propositions 4.1.6 and 4.2.3, we obtain the functoriality of $R \Gamma_{c}(\cdot)$ with respect to proper maps.

Definition 5.3.2. Let $\mathscr{L}$ be a smooth algebraic scheme over $\mathscr{V}$. For any integers $n$ and $i$, we define the absolute cohomology groups of $\mathscr{X}$ as

$$
H_{\mathrm{abs}}^{n}(\mathscr{X}, i):=\operatorname{Hom}_{p H D}(\mathbb{K}, R \Gamma(\mathscr{X})(i)[n])=H^{n}(\Gamma(\mathbb{K}, R \Gamma(\mathscr{X})(i)))
$$

and the absolute cohomology with compact support groups of $\mathscr{X}$ as

$$
H_{\mathrm{abs}, c}^{n}(\mathscr{X}, i):=\operatorname{Hom}_{p H D}\left(\mathbb{K}, R \Gamma_{c}(\mathscr{X})(i)[n]\right)=H^{n}\left(\Gamma\left(\mathbb{K}, R \Gamma_{c}(\mathscr{X})(i)\right)\right) .
$$

A direct consequence of the definition is the existence of the following long exact sequence, which should be considered to be a $p$-adic analog of the corresponding sequence for Deligne-Beilinson cohomology [Bey̆linson 1985, Introduction].

Proposition 5.3.3. With the notation above, we have the long exact sequences

$$
\rightarrow H_{\mathrm{abs}}^{n}(\mathscr{X}, i) \rightarrow H_{\mathrm{rig}}^{n}\left(\mathscr{X}_{k} / K_{0}\right) \oplus F^{i} H_{\mathrm{dR}}^{n}\left(\mathscr{X}_{K}\right) \stackrel{h}{\rightarrow} H_{\mathrm{rig}}^{n}\left(\mathscr{X}_{k} / K_{0}\right) \oplus H_{\mathrm{rig}}^{n}\left(\mathscr{X}_{k} / K\right) \stackrel{+}{\rightarrow},
$$

where $h\left(x_{0}, x_{\mathrm{dR}}\right):=\left(\phi\left(x_{0}^{\sigma}\right)-p^{i} x_{0}, x_{0} \otimes 1_{K}-s p\left(x_{\mathrm{dR}}\right)\right)$ and

$\rightarrow H_{\mathrm{abs}, c}^{n}(\mathscr{X}, i) \rightarrow H_{\mathrm{rig}, c}^{n}\left(\mathscr{X}_{k} / K_{0}\right) \oplus F^{i} H_{\mathrm{dR}, c}^{n}\left(\mathscr{X}_{K}\right) \stackrel{h_{c}}{\rightarrow} H_{\mathrm{rig}, c}^{n}\left(\mathscr{X}_{k} / K_{0}\right) \oplus H_{\mathrm{dR}, c}^{n}\left(\mathscr{X}_{K}\right) \stackrel{+}{\rightarrow}$,

where $h_{c}\left(x_{0}, x_{\mathrm{dR}}\right):=\left(\phi_{c}\left(x_{0} \sigma\right)-p^{i} x_{0}, \cos p\left(x_{0} \otimes 1_{K}\right)-x_{\mathrm{dR}}\right)$.

Proof. By Proposition 2.2.2, the absolute cohomology is the cohomology of a mapping cone, namely $\Gamma(\mathbb{K}, R \Gamma(\mathscr{C}))$ (or $\Gamma_{c}(\mathbb{K}, R \Gamma(\mathscr{C})$ ) for the compact support case). The long exact sequences above are easily induced from the distinguished triangle defined by the term of the mapping cone (see Remark 2.2.1(iii)). Indeed, one need only note that the map $c: R \Gamma_{\text {rig }}\left(\mathscr{X} / K_{0}\right) \otimes K \rightarrow R \Gamma_{K}(\mathscr{X})\left(\right.$ resp. $s: R \Gamma_{\mathrm{dR}}(\mathscr{X}) \rightarrow R \Gamma_{K}(\mathscr{X})$ ) is a quasi-isomorphism. 
Proposition 5.3.4. With the notation above, there is a canonical isomorphism between the absolute cohomology we have defined and the (rigid) syntomic cohomology of Besser:

$$
H_{\mathrm{syn}}^{n}(\mathscr{X}, i) \cong H_{\mathrm{abs}}^{n}(\mathscr{X}, i) .
$$

Proof. Besser defines a complex

$\mathbb{R} \Gamma_{\text {Bes }}(\mathscr{X}, i)$

$$
:=\operatorname{Cone}\left(\mathbb{R} \Gamma_{\text {rig }}\left(\mathscr{X} / K_{0}\right) \oplus F i l^{i} \mathbb{R} \Gamma_{\mathrm{dR}}(\mathscr{X}) \rightarrow \mathbb{R} \Gamma_{\text {rig }}\left(\mathscr{X} / K_{0}\right) \oplus \mathbb{R} \Gamma_{\text {rig }}(\mathscr{X} / K)\right)[-1]
$$

and the syntomic cohomology groups (of degree $n$ and twisted $i) H^{n}\left(\mathbb{R} \Gamma_{\mathrm{Bes}}(\mathscr{X}, i)\right.$ ) (see [Besser 2000, proof of Proposition 6.3]). Note that, modulo the choice of the flasque resolution, $\mathbb{R} \Gamma_{\text {rig }}\left(\mathscr{X} / K_{0}\right)=R \Gamma_{\text {rig }}\left(\mathscr{X} / K_{0}\right)$ (the left-hand side is the notation used by Besser with the bold $\mathbb{R})$; the complex $F i l^{i} \mathbb{R} \Gamma_{\mathrm{dR}}(\mathscr{C})$ is a direct limit over all the normal crossing compactifications of $\mathscr{X}_{K}$ of complexes of the form $\Gamma\left(Y, \mathrm{Gd}_{P t(Y)}^{2} \sigma^{\geq i} \Omega_{Y}\langle D\rangle\right)$ so that our $F^{i} R \Gamma_{\mathrm{dR}}(\mathscr{X})$ is an element of this direct limit. To conclude the proof, recall that by Remark 2.2.1(iii), we obtain

$$
\begin{aligned}
& H_{\mathrm{abs}}^{n}(\mathscr{X}, i) \\
& \cong H^{n}\left(\operatorname{Cone}\left(R \Gamma_{\text {rig }}\left(\mathscr{X} / K_{0}\right) \oplus F^{i} R \Gamma_{\mathrm{dR}}(\mathscr{X}) \rightarrow R \Gamma_{\text {rig }}\left(\mathscr{X} / K_{0}\right) \oplus R \Gamma_{\text {rig }}(\mathscr{X} / K)\right)[-1]\right),
\end{aligned}
$$

and it is easy to check that the maps in the two mapping cones are defined in the same way.

Remark 5.3.5. The isomorphism $H^{n}\left(R \Gamma_{\text {syn }}(\mathscr{X}, i)\right) \cong \operatorname{Hom}_{p H D}(\mathbb{K}, R \Gamma(\mathscr{X})(i)[n])$ can be viewed as a generalization of the result of Bannai [2002], who considers only smooth schemes $\mathscr{X}$ with a fixed compactification $\mathscr{Y}$ and such that $\mathscr{Y} \backslash \mathscr{X}=\mathscr{D}$ is a relative normal crossings divisor over $\mathscr{V}$. Moreover, Bannai's construction is not functorial with respect to $\mathscr{X}$; functoriality holds only with respect to a socalled syntomic datum. We should point out that the category defined by Bannai is endowed with a $t$-structure whose heart is the category $M F_{K}^{f}$ of (weakly) admissible filtered Frobenius modules. In our case, we don't have such a nice picture.

Remark 5.3.6. The category of $p$-adic Hodge complexes is not endowed with internal Hom. This is due to the fact that the Frobenius is only a quasi-isomorphism; hence, we cannot invert it. In particular, this happens for the complex $R \Gamma_{c}(\mathscr{X})$; thus, we cannot define the dual $R \Gamma_{c}(\mathscr{X})^{\vee}:=\mathbb{R} \mathscr{H} \operatorname{om}\left(R \Gamma_{c}(\mathscr{X}), \mathbb{K}\right)$ and an absolute homology theory as

$$
H_{n}^{\mathrm{abs}}(\mathscr{Q}, i):=\operatorname{Hom}_{p H D}\left(\mathbb{K}, R \Gamma_{c}(\mathscr{X})^{\vee}(-i)[-n]\right) ;
$$

see [Huber 1995, Section 15.3]. Nevertheless, the usual adjunction between Hom and $\otimes$ should give a natural isomorphism

$$
\operatorname{Hom}_{p H D}\left(\mathbb{K}, R \Gamma_{c}(\mathscr{X})^{\vee}\right) \cong \operatorname{Hom}_{p H D}\left(R \Gamma_{c}(\mathscr{X}), \mathbb{K}\right),
$$


and the right term does makes sense in our setting. This motivates the following definition:

Definition 5.3.7. With the notation of Definition 5.3.2, we define the absolute homology groups of $\mathscr{X}$ as

$$
H_{n}^{\mathrm{abs}}(\mathscr{X}, i):=\operatorname{Hom}_{p H D}\left(R \Gamma_{c}(\mathscr{X}), \mathbb{K}(-i)[-n]\right)=H^{-n}\left(\Gamma\left(R \Gamma_{c}(\mathscr{X}), \mathbb{K}(-i)\right)\right) .
$$

5.4. Cup product and Gysin map. We are going to prove that there is a morphism

$$
R \Gamma(\mathscr{X}) \otimes R \Gamma_{c}(\mathscr{C}) \rightarrow R \Gamma_{c}(\mathscr{X})
$$

of $p$-adic Hodge complexes. This induces a pairing at the level of the complexes computing absolute cohomology. The key point is the compatibility of the de Rham and rigid pairings with respect to the specialization and cospecialization maps.

Let us start by fixing some notation. Let $\mathscr{X}$ be a smooth algebraic $\mathscr{V}$-scheme, $g: \mathscr{X} \rightarrow \mathscr{Y}$ be a gncd compactification and $\mathscr{D}=\mathscr{Y} \backslash \mathscr{X}$ be the complement; $\mathscr{Y}$ is the rigid analytic space associated to the $K$-scheme $\mathscr{Y}_{K}$; as before, we let $w: \mathscr{Y} \rightarrow \mathscr{Y}_{K \text {,zar }}$ denote the canonical morphism of sites (see the notation after Proposition 5.1.2). For any $\mathrm{Og}_{K}$-module $F, w^{*}$ denotes its pullback.

\section{Remark 5.4.1.}

(i) The wedge product of algebraic differentials induces the pairing

$$
p_{\mathrm{dR}}: \Omega_{\mathrm{g}_{K}}\left\langle\mathscr{D}_{K}\right\rangle \otimes I \Omega_{\mathrm{g}_{K}}\left\langle\mathscr{D}_{K}\right\rangle \rightarrow I \Omega \mathrm{g}_{K}\left\langle\mathscr{D}_{K}\right\rangle .
$$

(ii) The analytification of $p_{\mathrm{dR}}$ gives a pairing

$$
w^{*} \Omega \mathscr{y}_{K}\left\langle\mathscr{D}_{K}\right\rangle \otimes w^{*} I \Omega \mathscr{y}_{K}\left\langle\mathscr{D}_{K}\right\rangle \rightarrow w^{*} I \Omega \mathscr{g}_{K}\left\langle\mathscr{D}_{K}\right\rangle .
$$

Hence, by [Berthelot 1997a, Lemma 2.1], we get the pairing

$$
p_{\text {rig }}: j^{\dagger} w^{*} \Omega_{\mathscr{g}_{K}}\left\langle\mathscr{D}_{K}\right\rangle \otimes \underline{\Gamma}_{\mathscr{X}_{k}[} w^{*} I \Omega_{\mathscr{g}_{K}}\left\langle\mathscr{D}_{K}\right\rangle \rightarrow \underline{\Gamma}_{\mathscr{x}_{k}[} w^{*} I \Omega_{\mathscr{g}_{K}}\left\langle\mathscr{D}_{K}\right\rangle .
$$

Lemma 5.4.2 (sheaves level). The diagram

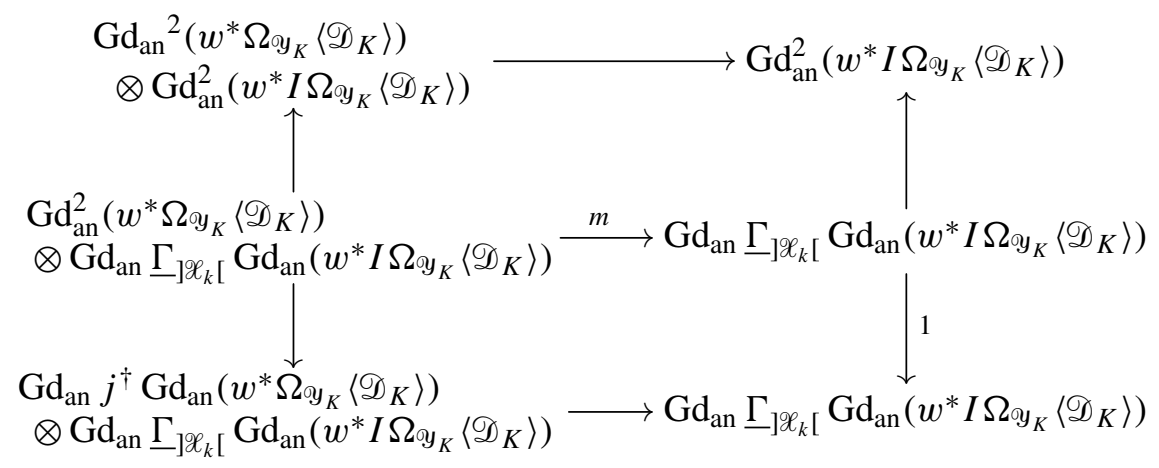

commutes, where $m:=p_{\text {rig }} \circ\left(j^{\dagger} \otimes 1\right)$ by definition. 
Proof. The bottom square commutes by construction, and we get $p_{\text {rig }} \circ\left(j^{\dagger} \otimes 1\right)=$ $w^{*} p_{\mathrm{dR}}$ restricted to $w^{*} \Omega_{\mathrm{g}_{K}}\left\langle\mathscr{D}_{K}\right\rangle \otimes \underline{\Gamma}_{] \mathscr{X}_{k}[} w^{*} I \Omega_{\mathrm{g}_{K}}\left\langle\mathscr{D}_{K}\right\rangle$.

Proposition 5.4.3. Let $\mathscr{X}$ be a smooth $\mathscr{V}$-scheme. Then there exists a morphism

$$
\pi: R \Gamma(\mathscr{X}) \otimes R \Gamma_{c}(\mathscr{X}) \rightarrow R \Gamma_{c}(\mathscr{X})
$$

of p-adic Hodge complexes, which is functorial with respect to $\mathscr{L}$ (as a morphism in pHD). Moreover, taking the cohomology of this map, we get the compatibility

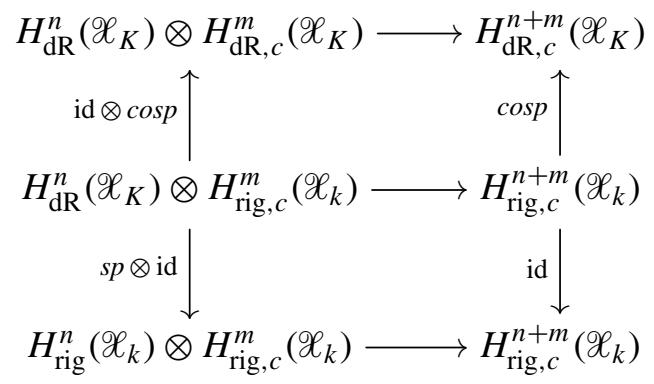

Proof. It is sufficient to provide a pairing of the enlarged diagrams (see Remark 2.2.4). Thus, we have to define a morphism of diagrams $\pi^{\prime}: R \Gamma^{\prime}(\mathscr{X}) \otimes R \Gamma_{c}^{\prime}(\mathscr{X}) \rightarrow R \Gamma_{c}^{\prime}(\mathscr{X})$ (notation as in Section 5.3). It is easy to construct $\pi^{\prime}$ using the previous lemma and the compatibility of the Godement resolution with the tensor product.

Corollary 5.4.4. There is a functorial pairing (induced by $\pi$ of Proposition 5.4.3)

$$
H_{\mathrm{abs}}^{n}(\mathscr{X}, i) \otimes H_{\mathrm{abs}, c}^{m}(\mathscr{X}, j) \rightarrow H_{\mathrm{abs}, c}^{n+m}(\mathscr{X}, i+j) .
$$

Proof. Consider the pairing of Proposition 5.4.3, which induces a morphism $R \Gamma(\mathscr{X})(i) \otimes R \Gamma_{c}(\mathscr{X})(j) \rightarrow R \Gamma_{c}(\mathscr{X})(i+j)$. We then get the corollary by Lemma 2.2.3 and Definition 5.3.2.

Proposition 5.4.5 (Poincaré duality). Let $\mathscr{X}$ be a smooth and algebraic $\mathscr{V}$-scheme of dimension $d$. Then there is a canonical isomorphism

$$
H_{\mathrm{abs}}^{n}(\mathscr{X}, i) \cong H_{2 d-n}^{\mathrm{abs}}(\mathscr{X}, d-i) .
$$

Proof. By definition (Equations (4) and (6)), it is sufficient to prove that the complex $\Gamma(\mathbb{K}, R \Gamma(\mathscr{X}))$ is quasi-isomorphic to the diagram $\Gamma\left(R \Gamma_{c}(\mathscr{X}), \mathbb{K}[-2 d](-d)\right)$.

First recall that $\Gamma(\mathbb{K}, R \Gamma(\mathscr{X}))$ is defined as

Cone $\left(R \Gamma_{\text {rig }}\left(\mathscr{X} / K_{0}\right) \oplus R \Gamma_{K}(\mathscr{X}) \oplus F^{0} R \Gamma_{\mathrm{dR}}(\mathscr{X})\right.$

$$
\left.\stackrel{\psi}{\rightarrow} R \Gamma_{\text {rig }}\left(\mathscr{X} / K_{0}\right) \oplus R \Gamma_{K}(\mathscr{X}) \oplus R \Gamma_{K}(\mathscr{X})\right)[-1],
$$


where $\psi\left(x_{0}, x_{K}, x_{\mathrm{dR}}\right):=\left(\phi\left(x_{0}\right)-x_{0}, c\left(x_{0} \otimes \mathrm{id}_{K}\right)-x_{K}, x_{K}-s\left(x_{\mathrm{dR}}\right)\right)$. To define the desired map, we need to modify this complex, replacing $R \Gamma_{K}(\mathscr{X})$ with $R \Gamma_{\mathrm{dR}}(\mathscr{X})$ as

Cone $\left(R \Gamma_{\text {rig }}\left(\mathscr{X} / K_{0}\right) \oplus R \Gamma_{\mathrm{dR}}(\mathscr{C}) \oplus F^{0} R \Gamma_{\mathrm{dR}}(\mathscr{X})\right.$

$$
\left.\stackrel{\psi^{\prime}}{\rightarrow} R \Gamma_{\mathrm{rig}}\left(\mathscr{L} / K_{0}\right) \oplus R \Gamma_{K}(\mathscr{X}) \oplus R \Gamma_{\mathrm{dR}}(\mathscr{X})\right)[-1],
$$

where $\psi^{\prime}\left(x_{0}, x_{\mathrm{dR}}^{\prime}, x_{\mathrm{dR}}\right):=\left(\phi\left(x_{0}\right)-x_{0}, c\left(x_{0} \otimes \mathrm{id}_{K}\right)-s\left(x_{\mathrm{dR}}^{\prime}\right), x_{\mathrm{dR}}^{\prime}-x_{\mathrm{dR}}\right)$. It is easy to see that this new complex, call it $M^{\bullet}$, is quasi-isomorphic to $\Gamma(\mathbb{K}, R \Gamma(\mathscr{X}))$.

Because the filtered complex $R \Gamma_{\mathrm{dR}, c}(\mathscr{X})$ is strict, the truncation $\tau_{\geq 2 d} R \Gamma_{\mathrm{dR}, c}(\mathscr{C})$ is the usual truncation of complexes of $K$-vector spaces (see Lemma 2.1.1(iii) and Remark 2.0.10). Then the cup product induces a morphism of complexes $M^{\bullet} \rightarrow \Gamma\left(R \Gamma_{c}(\mathscr{X}), \tau_{\geq 2 d} R \Gamma_{c}(\mathscr{X})\right)$ that is a quasi-isomorphism by the Poincaré duality theorems for rigid and de Rham cohomology [Berthelot 1997a; Huber 1995]. Explicitly, this map is induced by the commutative diagram

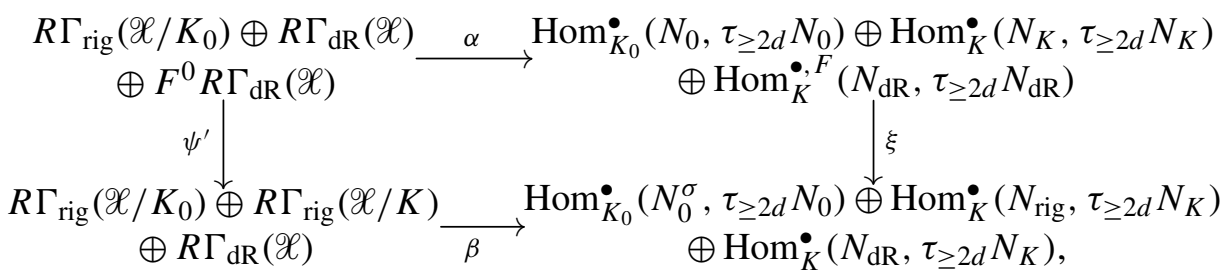

where $N:=R \Gamma_{c}(\mathscr{X})$

$\xi\left(f_{0}, f_{K}, \mathrm{dR}\right):=\left(\phi_{c} \circ f_{0}^{\sigma}-f_{0} \circ \phi_{c}, c \circ\left(f_{0} \otimes \mathrm{id}_{K}\right)-f_{K} \circ c, f_{K} \circ s-s \circ x_{\mathrm{dR}}\right)$, $\alpha\left(x_{0}, x_{\mathrm{dR}}^{\prime}, x_{\mathrm{dR}}\right):\left(y_{0}, y_{K}, y_{\mathrm{dR}}\right) \mapsto\left(x_{0} \cup y_{0}, s\left(x_{\mathrm{dR}}^{\prime}\right) \cup y_{K}, x_{\mathrm{dR}} \cup y_{\mathrm{dR}}\right)$, $\beta\left(x_{0}, x_{\text {rig }}, x_{\mathrm{dR}}\right):\left(y_{0}, y_{\text {rig }}, y_{\mathrm{dR}}\right) \mapsto\left(x_{0} \cup \phi_{c}\left(y_{0}\right), x_{\text {rig }} \cup y_{\text {rig }}, x_{\mathrm{dR}} \cup y_{\mathrm{dR}}\right)$.

To conclude the proof, it is sufficient to apply the exact functor $\Gamma\left(R \Gamma_{c}(\mathscr{X}), \cdot\right)$ to the quasi-isomorphisms

$$
\tau_{\geq 2 d} R \Gamma_{c}(\mathscr{X}) \leftarrow H^{2 d}\left(R \Gamma_{c}(\mathscr{X})\right)[-2 d] \rightarrow \mathbb{K}(-d)[-2 d] .
$$

Remark 5.4.6. We would like to point out some technical issues regarding Poincaré duality in syntomic cohomology.

(i) If it were possible to define an internal Hom in the category $p H C$ of $p$-adic Hodge complexes (see Remark 5.3.6), then by Proposition 5.4.3, one would obtain the natural isomorphism 
in the triangulated category $p H D$, where $R \Gamma_{c}(\mathscr{X})^{\vee}:=\mathbb{R} \mathscr{H} \operatorname{mom}_{p H C}\left(R \Gamma_{c}(\mathscr{X}), \mathbb{K}\right)$ is the dual of $R \Gamma_{c}(\mathscr{X})$. Then one would get by adjunction the duality

$$
\begin{aligned}
& \operatorname{Hom}_{p H D}(\mathbb{K}, R \Gamma(X)(i)[n]) \cong \operatorname{Hom}_{p H D}\left(\mathbb{K}, R \Gamma_{c}(X)^{\vee}(i-d)[n-2 d]\right) \\
& \cong \operatorname{Hom}_{p H D}\left(R \Gamma_{c}(X)(d-i)[2 d-n], \mathbb{K}\right) .
\end{aligned}
$$

(ii) The Grothendieck-Leray spectral sequence for absolute homology is

$$
E_{2}^{p, q}=\operatorname{Hom}_{p H D}\left(H^{-q}\left(R \Gamma_{c}(\mathscr{X})(i)\right)[-p], \mathbb{K}\right),
$$

and it degenerates to the short exact sequences

$0 \rightarrow \operatorname{Hom}_{p H D}\left(H^{n}\left(R \Gamma_{c}(\mathscr{L})(i)\right), \mathbb{K}\right) \rightarrow H_{n}^{\mathrm{syn}}(\mathscr{X}, i)$

$$
\rightarrow \operatorname{Hom}_{p H D}\left(H^{n+1}\left(R \Gamma_{c}(\mathscr{Q})(i)\right), \mathbb{K}[1]\right) \rightarrow 0 .
$$

Directly by Proposition 2.2.2, the group $\operatorname{Hom}_{p H D}\left(H^{n}\left(R \Gamma_{c}(\mathscr{X})(i)\right)\right.$, $\left.\mathbb{K}\right)$ is

$$
\begin{aligned}
\left\{\left(x_{0}, x_{\mathrm{dR}}\right) \in H_{\mathrm{rig}, c}^{n}\left(\mathscr{X}_{k} / K_{0}\right)^{\vee} \oplus\left(H_{\mathrm{dR}, c}^{n}\left(\mathscr{X}_{K}\right) / F^{i+1}\right)^{\vee}\right. & \\
& \left.:\left(x_{0} \otimes 1_{K}\right)=x_{\mathrm{dR}} \circ \cos p, x_{0} \circ \phi_{c}=p^{i} x_{0}^{\sigma}\right\} .
\end{aligned}
$$

In cohomology, the Frobenius is an invertible; hence, the equation $x_{0} \circ \phi_{c}=p^{i} x_{0}^{\sigma}$ is equivalent to $x_{0}=p^{i} x_{0}^{\sigma} \phi_{c}^{-1}$, where the latter is the formula for the Frobenius of internal $\mathscr{H o m}\left(H_{\text {rig }, c}^{n}\left(\mathscr{L}_{k}\right), K(-i)\right)$ in the category of isocrystals (that is, modules with Frobenius). Hence, by Poincaré duality, we get $\phi\left(\left(x_{0}^{\vee}\right)^{\sigma}\right)=p^{d-i} x_{0}^{\vee}$; $x^{\vee} \in H_{\text {rig }}^{2 d-n}\left(\mathscr{X}_{k} / K_{0}\right)$ is the cohomology class corresponding to $x_{0}$, where $\phi$ is the Frobenius of $H_{\text {rig }}^{2 d-n}\left(\mathscr{X}_{k} / K_{0}\right)$.

(iii) The absolute homology is defined via the complex $R \Gamma_{c}(\mathscr{X})$, but it is not clear how to relate it to the dual of the absolute cohomology with compact support.

Corollary 5.4.7 (Gysin map). Let $f: \mathscr{L} \rightarrow$ y be a proper morphism of smooth algebraic $\mathcal{V}$-schemes of relative dimension $d$ and $e$, respectively. Then there is a canonical map

$$
f_{*}: H_{\mathrm{abs}}^{n}(\mathscr{X}, i) \rightarrow H_{\mathrm{abs}}^{n+2 c}(\mathscr{Y}, i+c),
$$

where $c=e-d$.

Proof. This is a direct consequence of the previous proposition and the functoriality of $R \Gamma_{c}(\mathscr{X})$ with respect to proper morphisms.

Remark 5.4.8. With the notation above, we get a morphism of spectral sequences

$$
\begin{aligned}
g: E_{2}^{p, q}(\mathscr{X}):=\operatorname{Hom}_{p H D}\left(\mathbb{K}, H^{q}(\mathscr{X})(i)[p]\right) \rightarrow E_{2}^{p, q+2 c}(\mathscr{Y}) \\
\quad:=\operatorname{Hom}_{p H D}\left(\mathbb{K}, H^{q+2 c}(\mathscr{Y})(i)[p]\right)
\end{aligned}
$$

compatible with $f_{*}$. The map $g$ is induced by the Gysin morphism in de Rham and rigid cohomology. 
We conclude by saying that it is natural to expect that the Gysin map for syntomic cohomology is compatible with the $K$-theory pushforward under the regulator defined by Besser. We plan to address this open problem in a future work.

\section{Acknowledgment}

We acknowledge the help of and useful discussion with K. Bannai, A. Besser, F. Ivorra and Q. Liu.

\section{References}

[Artin et al. 1972a] M. Artin, A. Grothendieck, J. Verdier, P. Deligne, and B. Saint-Donat (editors), Théorie des topos et cohomologie étale des schémas. Tome 1: Théorie des topos, Lecture Notes in Mathematics 269, Springer, Berlin, 1972. Séminaire de Géométrie Algébrique du Bois-Marie 1963-1964. MR 50 \#7130 Zbl 0234.00007

[Artin et al. 1972b] M. Artin, A. Grothendieck, J. Verdier, P. Deligne, and B. Saint-Donat (editors), Théorie des topos et cohomologie étale des schémas. Tome 2, Lecture Notes in Mathematics 270 , Springer, Berlin, 1972. Séminaire de Géométrie Algébrique du Bois-Marie 1963-1964. MR 50 \#7131 Zbl 0237.00012

[Baldassarri et al. 2004] F. Baldassarri, M. Cailotto, and L. Fiorot, "Poincaré duality for algebraic de Rham cohomology", Manuscripta Math. 114:1 (2004), 61-116. MR 2006b:14035 Zbl 1071.14023

[Bannai 2002] K. Bannai, "Syntomic cohomology as a p-adic absolute Hodge cohomology", Math. Z. 242:3 (2002), 443-480. MR 2004e:14037 Zbl 1053.14018

[Beǔlinson 1985] A. A. Beŭlinson, "Higher regulators and values of $L$-functions", J. Sov. Math. 30 (1985), 2036-2070. Zbl 0588.14013

[Běllinson 1986] A. A. Be1̌linson, "Notes on absolute Hodge cohomology", pp. 35-68 in Applications of algebraic $K$-theory to algebraic geometry and number theory, Part I (Boulder, CO, 1983), edited by S. J. Bloch et al., Contemp. Math. 55, Amer. Math. Soc., Providence, RI, 1986. MR 87m:14019 Zbl 0621.14011

[Berthelot 1997a] P. Berthelot, "Dualité de Poincaré et formule de Künneth en cohomologie rigide", C. R. Acad. Sci. Paris Sér. I Math. 325:5 (1997), 493-498. MR 2000c:14023 Zbl 0908.14006

[Berthelot 1997b] P. Berthelot, "Finitude et pureté cohomologique en cohomologie rigide", Invent. Math. 128:2 (1997), 329-377. MR 98j:14023 Zbl 0908.14005

[Berthelot et al. 1971] P. Berthelot, A. Grothendieck, and L. Illusie (editors), Théorie des intersections et théorème de Riemann-Roch (SGA 6), Lecture Notes in Mathematics 225, Springer, Berlin, 1971. MR 50 \#7133 Zbl 0218.14001

[Besser 2000] A. Besser, "Syntomic regulators and p-adic integration, I: Rigid syntomic regulators", Israel J. Math. 120 (2000), 291-334. MR 2002c:14035 Zbl 1001.19003

[Bloch 1986] S. Bloch, "Algebraic cycles and higher K-theory", Adv. in Math. 61:3 (1986), 267-304. MR 88f:18010 Zbl 0608.14004

[Bosch et al. 1995] S. Bosch, W. Lütkebohmert, and M. Raynaud, "Formal and rigid geometry, IV: The reduced fibre theorem”, Invent. Math. 119:2 (1995), 361-398. MR 97e:11075 Zbl 0839.14014 [Conrad 2007] B. Conrad, "Deligne's notes on Nagata compactifications", J. Ramanujan Math. Soc. 22:3 (2007), 205-257. MR 2009d:14002 Zbl 1142.14001

[Deligne 1971] P. Deligne, “Théorie de Hodge, II”, Inst. Hautes Études Sci. Publ. Math. 40 (1971), 5-57. MR 58 \#16653a Zbl 0219.14007 
[Deligne and Illusie 1987] P. Deligne and L. Illusie, "Relèvements modulo $p^{2}$ et décomposition du complexe de de Rham”, Invent. Math. 89:2 (1987), 247-270. MR 88j:14029 Zbl 0632.14017

[Friedlander and Suslin 2002] E. M. Friedlander and A. Suslin, "The spectral sequence relating algebraic K-theory to motivic cohomology", Ann. Sci. École Norm. Sup. (4) 35:6 (2002), 773-875. MR 2004b:19006 Zbl 1047.14011

[GAGA 1955-1956] J.-P. Serre, "Géométrie algébrique et géométrie analytique", Ann. Inst. Fourier, Grenoble 6 (1955-1956), 1-42. MR 18,511a Zbl 0075.30401

[Gillet and Messing 1987] H. Gillet and W. Messing, "Cycle classes and Riemann-Roch for crystalline cohomology”, Duke Math. J. 55:3 (1987), 501-538. MR 89c:14025 Zbl 0651.14014

[Grothendieck 1966a] A. Grothendieck, "Étude locale des schémas et des morphismes de schémas, III", Inst. Hautes Études Sci. Publ. Math. 28 (1966), 255. MR 36 \#178 Zbl 0144.19904

[Grothendieck 1966b] A. Grothendieck, "On the de Rham cohomology of algebraic varieties", Inst. Hautes Études Sci. Publ. Math. 29 (1966), 95-103. MR 33 \#7343 Zbl 0145.17602

[Grothendieck 1967] A. Grothendieck, "Étude locale des schémas et des morphismes de schémas, IV”, Inst. Hautes Études Sci. Publ. Math. 32 (1967), 361. MR 39 \#220 Zbl 0153.22301

[Hartshorne 1975] R. Hartshorne, "On the De Rham cohomology of algebraic varieties", Inst. Hautes Études Sci. Publ. Math. 45 (1975), 5-99. MR 55 \#5633 Zbl 0326.14004

[Hartshorne 1977] R. Hartshorne, Algebraic geometry, Graduate Texts in Mathematics 52, Springer, New York, 1977. MR 57 \#3116 Zbl 0367.14001

[Huber 1995] A. Huber, Mixed motives and their realization in derived categories, Lecture Notes in Mathematics 1604, Springer, Berlin, 1995. MR 98d:14030 Zbl 0938.14008

[Ivorra 2005] F. Ivorra, Réalisation l-adique des motifs mixtes, thesis, Université Paris, 6, 2005, Available at http://www.uni-due.de/ hm0035/These.pdf.

[Jannsen 1990] U. Jannsen, Mixed motives and algebraic K-theory, Lecture Notes in Mathematics 1400, Springer, Berlin, 1990. MR 91g:14008 Zbl 0691.14001

[Le Stum 2007] B. Le Stum, Rigid cohomology, Cambridge Tracts in Mathematics 172, Cambridge University Press, 2007. MR 2009c:14029 Zbl 1131.14001

[Levine 1998] M. Levine, Mixed motives, Mathematical Surveys and Monographs 57, American Mathematical Society, Providence, RI, 1998. MR 99i:14025 Zbl 0902.14003

[Mazza et al. 2006] C. Mazza, V. Voevodsky, and C. Weibel, Lecture notes on motivic cohomology, Clay Mathematics Monographs 2, American Mathematical Society, Providence, RI, 2006. MR 2007e:14035 Zbl 1115.14010

[Peters and Steenbrink 2008] C. A. M. Peters and J. H. M. Steenbrink, Mixed Hodge structures, Ergebnisse der Mathematik und ihrer Grenzgebiete (3) 52, Springer, Berlin, 2008. MR 2009c:14018 Zbl 1138.14002

[Petrequin 2003] D. Petrequin, "Classes de Chern et classes de cycles en cohomologie rigide", Bull. Soc. Math. France 131:1 (2003), 59-121. MR 2004b:14030 Zbl 1083.14505

[van der Put and Schneider 1995] M. van der Put and P. Schneider, "Points and topologies in rigid geometry", Math. Ann. 302:1 (1995), 81-103. MR 96k:32070 Zbl 0867.11049

[Suslin and Voevodsky 2000] A. Suslin and V. Voevodsky, "Relative cycles and Chow sheaves", pp. 10-86 in Cycles, transfers, and motivic homology theories, Ann. of Math. Stud. 143, Princeton Univ. Press, 2000. MR 1764199 Zbl 1019.14004

[Weibel 1994] C. A. Weibel, An introduction to homological algebra, Cambridge Studies in Advanced Mathematics 38, Cambridge University Press, 1994. MR 95f:18001 Zbl 0797.18001

[Włodarczyk 2005] J. Włodarczyk, "Simple Hironaka resolution in characteristic zero", J. Amer. Math. Soc. 18:4 (2005), 779-822. MR 2006f:14014 Zbl 1084.14018 
Communicated by Hélène Esnault

Received 2010-10-12 Revised 2011-12-22 Accepted 2012-05-03

chiarbru@math.unipd.it

Università degli Studi di Padova, Via Trieste 63, 35100 Padova, Italy

ciccioni@math.unipd.it Università degli Studi di Padova, Via Trieste 63, 35100 Padova, Italy

nicola.mazzari@math.u-bordeaux1.fr

IMB, Université Bordeaux 1 351, cours de la Libération, 33405 Talence cedex, France 


\section{Algebra \& Number Theory}

msp.org/ant

\section{EDITORS}

MANAGING EDITOR

Bjorn Poonen

Massachusetts Institute of Technology

Cambridge, USA

\author{
EDITORIAL BOARD CHAIR \\ David Eisenbud \\ University of California \\ Berkeley, USA
}

\section{BOARD OF EDITORS}

Georgia Benkart

Dave Benson

Richard E. Borcherds

John H. Coates

J-L. Colliot-Thélène

Brian D. Conrad

Hélène Esnault

Hubert Flenner

Edward Frenkel

Andrew Granville

Joseph Gubeladze

Ehud Hrushovski

Craig Huneke

Mikhail Kapranov

Yujiro Kawamata

János Kollár

Yuri Manin

Barry Mazur

Philippe Michel
University of Wisconsin, Madison, USA

University of Aberdeen, Scotland

University of California, Berkeley, USA

University of Cambridge, UK

CNRS, Université Paris-Sud, France

University of Michigan, USA

Freie Universität Berlin, Germany

Ruhr-Universität, Germany

University of California, Berkeley, USA

Université de Montréal, Canada

San Francisco State University, USA

Hebrew University, Israel

University of Virginia, USA

Yale University, USA

University of Tokyo, Japan

Princeton University, USA

Northwestern University, USA

Harvard University, USA

École Polytechnique Fédérale de Lausanne
Susan Montgomery

Shigefumi Mori

Raman Parimala

Jonathan Pila

Victor Reiner

Karl Rubin

Peter Sarnak

Joseph H. Silverman

Michael Singer

Vasudevan Srinivas

J. Toby Stafford

Bernd Sturmfels

Richard Taylor

Ravi Vakil

Michel van den Bergh

Marie-France Vignéras

Kei-Ichi Watanabe

Efim Zelmanov
University of Southern California, USA

RIMS, Kyoto University, Japan

Emory University, USA

University of Oxford, UK

University of Minnesota, USA

University of California, Irvine, USA

Princeton University, USA

Brown University, USA

North Carolina State University, USA

Tata Inst. of Fund. Research, India

University of Michigan, USA

University of California, Berkeley, USA

Harvard University, USA

Stanford University, USA

Hasselt University, Belgium

Université Paris VII, France

Nihon University, Japan

University of California, San Diego, USA

\section{PRODUCTION}

production@msp.org

Silvio Levy, Scientific Editor

See inside back cover or msp.org/ant for submission instructions.

The subscription price for 2013 is US \$200/year for the electronic version, and \$350/year ( $\$ 40$, if shipping outside the US) for print and electronic. Subscriptions, requests for back issues and changes of subscribers address should be sent to MSP.

Algebra \& Number Theory (ISSN 1944-7833 electronic, 1937-0652 printed) at Mathematical Sciences Publishers, 798 Evans Hall \#3840, c/o University of California, Berkeley, CA 94720-3840 is published continuously online. Periodical rate postage paid at Berkeley, CA 94704, and additional mailing offices.

ANT peer review and production are managed by EditFLOW ${ }^{\circledR}$ from Mathematical Sciences Publishers.

\section{PUBLISHED BY}

- mathematical sciences publishers

nonprofit scientific publishing

http://msp.org/

(C) 2013 Mathematical Sciences Publishers 


\section{Algebra \& Number Theory}

Volume $7 \quad$ No. $3 \quad 2013$

Ekedahl-Oort strata of hyperelliptic curves in characteristic 2

ARSEN ELKIN and RACHEL PRIES

Cycle classes and the syntomic regulator

Bruno Chiarellotto, Alice Ciccioni and Nicola Mazzari

Zeros of real irreducible characters of finite groups

SELENA MARINELli and PHAM HUU TIEP

The biHecke monoid of a finite Coxeter group and its representations

FLORENT HIVERT, ANNE SCHILLING and NICOLAS THIÉRY

Shuffle algebras, homology, and consecutive pattern avoidance

VLADIMIR DOTSENKO and ANTON KHOROSHKIN

701

Preperiodic points for families of polynomials

Dragos Ghioca, Liang-Chung Hsia and Thomas J. Tucker

$F$-blowups of normal surface singularities

NobUo HaRa, TADAKaZU SAWADA and TAKEHIKo YASUdA 